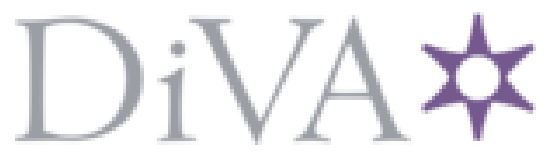

http://www.diva-portal.org

This is the published version of a paper published in Cochrane Database of Systematic Reviews.

Citation for the original published paper (version of record):

Byrne, M., Doherty, S., Fridlund, B., Mårtensson, J., Steinke, E E. et al. (2016)

Sexual counselling for sexual problems in patients with cardiovascular disease.

Cochrane Database of Systematic Reviews, (2): 1-39

http://dx.doi.org/10.1002/14651858.CD010988.pub2

Access to the published version may require subscription.

N.B. When citing this work, cite the original published paper.

Permanent link to this version:

http://urn.kb.se/resolve?urn=urn:nbn:se:hj:diva-29580 


\section{(A) Cochrane Library}

Cochrane Database of Systematic Reviews

\section{Sexual counselling for sexual problems in patients with} cardiovascular disease (Review)

Byrne M, Doherty S, Fridlund BGA, Mårtensson J, Steinke EE, Jaarsma T, Devane D

Byrne M, Doherty S, Fridlund BGA, Mårtensson J, Steinke EE, Jaarsma T, Devane D.

Sexual counselling for sexual problems in patients with cardiovascular disease.

Cochrane Database of Systematic Reviews 2016, Issue 2. Art. No.: CD010988.

DOI: 10.1002/14651858.CD010988.pub2.

www.cochranelibrary.com 
TABLE OF CONTENTS

HEADER . . . . . . . . . . . . . . . . . . . . . . . . . . . . . . . . . . . . 1

ABSTRACT . . . . . . . . . . . . . . . . . . . . . . . . . . . . . . . . . . . . . . . . . . . . . . .

PLAIN LANGUAGE SUMMARY . . . . . . . . . . . . . . . . . . . . . . . . . . . . . . . . . . . .

SUMMARY OF FINDINGS FOR THE MAIN COMPARISON $\quad$. . . . . . . . . . . . . . . . . . . . . . . . . . . 4

BACKGROUND . . . . . . . . . . . . . . . . . . . . . . . . . . . . . . . . . . . . 6

OBJECTIVES . . . . . . . . . . . . . . . . . . . . . . . . . . . . . . . . . . . . . . . . . . . . . .

METHODS . . . . . . . . . . . . . . . . . . . . . . . . . . . . . . . . . . . . . .

RESUlTS . . . . . . . . . . . . . . . . . . . . . . . . . . . . . . . . . . . . . 12

Figure 1. . . . . . . . . . . . . . . . . . . . . . . . . . . . . . . . . . . . 13

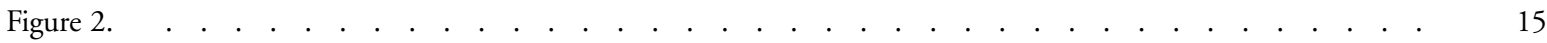

Figure $3 . \quad$. . . . . . . . . . . . . . . . . . . . . . . . . . . . . . . . . . . . . 16

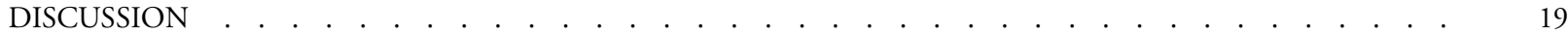

AUTHORS' CONCLUSIONS . . . . . . . . . . . . . . . . . . . . . . . . . . . . . . . . . . . .

ACKNOWLEDGEMENTS . . . . . . . . . . . . . . . . . . . . . . . . . . . . . . . . . . . . . . .

REFERENCES . . . . . . . . . . . . . . . . . . . . . . . . . . . . . . . . . . . . . . 21

CHARACTERISTICS OF STUDIES . . . . . . . . . . . . . . . . . . . . . . . . . . . . . . 26

DATA AND ANALYSES . . . . . . . . . . . . . . . . . . . . . . . . . . . . . . . . . . . . . . . . . . . . . . .

APPENDICES . . . . . . . . . . . . . . . . . . . . . . . . . . . . . . . . . . . . . . 34

CONTRIBUTIONS OF AUTHORS . . . . . . . . . . . . . . . . . . . . . . . . . . . . . . . . . . . . . . . . . .

DECLARATIONS OF INTEREST . . . . . . . . . . . . . . . . . . . . . . . . . . . . . . . . . . . . . . . .

SOURCES OF SUPPORT . . . . . . . . . . . . . . . . . . . . . . . . . . . . . . . . . . . . . . . . . . . . . . .

DIFFERENCES BETWEEN PROTOCOL AND REVIEW . . . . . . . . . . . . . . . . . . . . . . . . . 38

INDEX TERMS . . . . . . . . . . . . . . . . . . . . . . . . . . . . . . . . . . . . 39 


\title{
[Intervention Review] \\ Sexual counselling for sexual problems in patients with cardiovascular disease
}

\author{
Molly Byrne $^{1}$, Sally Doherty ${ }^{2}$, Bengt GA Fridlund ${ }^{3}$, Jan Mårtensson ${ }^{4}$, Elaine E Steinke ${ }^{5}$, Tiny Jaarsma ${ }^{6}$, Declan Devane ${ }^{7}$ \\ ${ }^{1}$ School of Psychology, National University of Ireland, Galway, Galway, Ireland. ${ }^{2}$ Department of Population and Health Science, School \\ of Psychology, RCSI, Dublin, Ireland. ${ }^{3}$ School of Health Sciences, Jönköping University, Jönköping, Sweden. ${ }^{4}$ Department of Nursing, \\ School of Health Sciences, Jönköping University, Jönköping, Sweden. ${ }^{5}$ School of Nursing, Wichita State University, Wichita, Kansas, \\ USA. ${ }^{6}$ Department of Social and Welfare Studies, University of Linköping, Norrköping, Sweden. ${ }^{7}$ School of Nursing and Midwifery, \\ National University of Ireland Galway, Galway, Ireland \\ Contact address: Molly Byrne, School of Psychology, National University of Ireland, Galway, St. Anthony's, Galway, County Galway, \\ Ireland. molly.byrne@nuigalway.ie.
}

Editorial group: Cochrane Heart Group.

Publication status and date: New, published in Issue 2, 2016.

Review content assessed as up-to-date: 2 March 2015.

Citation: Byrne M, Doherty S, Fridlund BGA, Mårtensson J, Steinke EE, Jaarsma T, Devane D. Sexual counselling for sexual problems in patients with cardiovascular disease. Cochrane Database of Systematic Reviews 2016, Issue 2. Art. No.: CD010988. DOI: 10.1002/14651858.CD010988.pub2.

Copyright (C) 2016 The Cochrane Collaboration. Published by John Wiley \& Sons, Ltd.

\begin{abstract}
A B S T R A C T
Background

Sexual problems are common among people with cardiovascular disease. Although clinical guidelines recommend sexual counselling for patients and their partners, there is little evidence on its effectiveness.

\section{Objectives}

To evaluate the effectiveness of sexual counselling interventions (in comparison to usual care) on sexuality-related outcomes in patients with cardiovascular disease and their partners.
\end{abstract}

\section{Search methods}

We searched CENTRAL, MEDLINE, EMBASE, and three other databases up to 2 March 2015 and two trials registers up to 3 February 2016.

\section{Selection criteria}

Randomised controlled trials (RCTs) and quasi-RCTs, including individual and cluster RCTs. We included studies that compared any intervention to counsel adult cardiac patients about sexual problems with usual care.

Data collection and analysis

We used standard methodological procedures expected by Cochrane.

\section{Main results}

We included three trials with 381 participants. We were unable to pool the data from the included studies due to the differences in interventions used; therefore we synthesised the trial findings narratively.

Sexual counselling for sexual problems in patients with cardiovascular disease (Review)

Copyright @ 2016 The Cochrane Collaboration. Published by John Wiley \& Sons, Ltd. 
Two trials were conducted in the USA and one was undertaken in Israel. All trials included participants who were admitted to hospital with myocardial infarction (MI), and one trial also included participants who had undergone coronary artery bypass grafting. All trials followed up participants for a minimum of three months post-intervention; the longest follow-up timepoint was five months.

One trial $(\mathrm{N}=92)$ tested an intensive (total five hours) psychotherapeutic sexual counselling intervention delivered by a sexual therapist. One trial $(\mathrm{N}=115)$ used a 15 -minute educational video plus written material on resuming sexual activity following a MI. One trial $(\mathrm{N}=174)$ tested the addition of a component that focused on resumption of sexual activity following a MI within a hospital cardiac rehabilitation programme.

The quality of the evidence for all outcomes was very low.

None of the included studies reported any outcomes from partners.

Two trials reported sexual function. One trial compared intervention and control groups on 12 separate sexual function subscales and used a repeated measures analysis of variance (ANOVA) test. They reported statistically significant differences in favour of the intervention. One trial compared intervention and control groups using a repeated measures analysis of covariance (ANCOVA), and concluded: "There were no significant differences between the two groups [for sexual function] at any of the time points".

Two trials reported sexual satisfaction. In one trial, the authors compared sexual satisfaction between intervention and control and used a repeated measured ANOVA; they reported "differences were reported in favour of the intervention". One trial compared intervention and control with a repeated measures ANCOVA and reported: "There were no significant differences between the two groups [for sexual satisfaction] at any of the timepoints".

All three included trials reported the number of patients returning to sexual activity following MI. One trial found some evidence of an effect of sexual counselling on reported rate of return to sexual activity (yes/no) at four months after completion of the intervention (relative risk (RR) 1.71, 95\% confidence interval (CI) 1.26 to 2.32; one trial, 92 participants, very low quality of evidence). Two trials found no evidence of an effect of sexual counselling on rate of return to sexual activity at 12 week (RR 1.01, 95\% CI 0.94 to 1.09 ; one trial, 127 participants, very low quality of evidence) and three month follow-up (RR 0.98, 95\% CI 0.88 to 1.10 ; one trial, 115 participants, very low quality of evidence).

Two trials reported psychological well-being. In one trial, no scores were reported, but the trial authors stated: "No treatment effects were observed on state anxiety as measured in three points in time". In the other trial no scores were reported but, based on results of a repeated measures ANCOVA to compare intervention and control groups, the trial authors stated: "The experimental group had significantly greater anxiety at one month post MI". They also reported: "There were no significant differences between the two groups [for anxiety] at any other time points".

One trial reporting relationship satisfaction and one trial reporting quality of life found no differences between intervention and control.

No trial reported on satisfaction in how sexual issues were addressed in cardiac rehabilitation services.

\section{Authors' conclusions}

We found no high quality evidence to support the effectiveness of sexual counselling for sexual problems in patients with cardiovascular disease. There is a clear need for robust, methodologically rigorous, adequately powered RCTs to test the effectiveness of sexual counselling interventions for people with cardiovascular disease and their partners.

\section{PLAIN LANGUAGE SUMMARY}

\section{Sexual counselling interventions for sexual problems in people with heart disease}

\section{Review question}

Are sexual counselling interventions helpful in reducing sexual problems for people with heart disease and their partners?

\section{Background}

People with heart disease are more likely than people without heart disease to report sexual problems. Sexual counselling for people with heart disease is when a health professional supports a person to safely return to sexual activity after their heart event, by giving them information and helping them to deal with their concerns and anxieties.

Sexual counselling for sexual problems in patients with cardiovascular disease (Review)

Copyright $\odot 2016$ The Cochrane Collaboration. Published by John Wiley \& Sons, Ltd. 


\section{Study characteristics}

We searched the international literature up to March 2015 for studies that compared any intervention designed to address and counsel people with heart disease in relation to sexual problems with usual care.

\section{Key results}

Three randomised controlled trials (clinical trials where people are allocated at random to one of two or more treatments) that included 381 participants in total met our inclusion criteria. The interventions tested in these studies were quite different from each other. All studies included people who had been admitted to hospital with a heart attack.

These studies do not provide strong evidence that sexual counselling can improve sexual outcomes for people with heart disease or their partners. One study, which reported the effects of an intensive intervention, involved five hours of sexual counselling provided by a psychotherapist. It reported improved sexual functioning and satisfaction, and reduced length of time taken for people to return to sexual activity following a cardiac event, in people that received the intervention compared to usual care. The other two studies reported no differences between people that received the intervention and usual care on these outcomes (both studies measured rate of return to sexual activity following a cardiac event; one of these two studies measured sexual functioning and satisfaction). There was no evidence that sexual counselling has an effect on quality of life (measured in one study) or marital satisfaction (measured in one study). One study found that patients who received a 15-minute sexual counselling educational video plus written material had higher levels of anxiety than usual care, as well as better knowledge about sex after a heart attack, one month after their cardiac event, but not at any other timepoints.

\section{Quality of the evidence}

The evidence was of very low quality. We judged the included studies to be at high risk of bias and study results were poorly reported. Bearing this in mind, the results of this review should be interpreted with caution. 
SUMMARY OF FINDINGS FOR THE MAIN COMPARISON [Explanation]

Sexual counselling compared with usual care for patients with cardiovascular disease

Participant or population: participants with cardiovascular disease

Setting: health services

Intervention: sexual counselling interventions

Comparison: usual care

\begin{tabular}{|c|c|c|c|}
\hline Outcomes & $\begin{array}{l}\text { Effect sexual counselling for } \\
\text { participants with cardiovas- } \\
\text { cular disease }\end{array}$ & $\begin{array}{l}\text { Number of participants } \\
\text { (studies) }\end{array}$ & $\begin{array}{l}\text { Quality of the evidence } \\
\text { (GRADE) }\end{array}$ \\
\hline Sexual function & $\begin{array}{l}\text { One study found higher lev- } \\
\text { els of sexual function (as- } \\
\text { sessed using the Sexual Func- } \\
\text { tion for Cardiac Patient Ques- } \\
\text { tionnaire) in the intervention } \\
\text { group in comparison to the } \\
\text { control group at one and } \\
\text { four month follow-up. Another } \\
\text { study found no difference in } \\
\text { sexual function (assessed us- } \\
\text { ing the Watts Sexual Func- } \\
\text { tion Questionnaire (WSFQ)) } \\
\text { between intervention and con- } \\
\text { trol groups at any timepoint }\end{array}$ & $\begin{array}{l}207 \\
\text { (2 RCTs) }\end{array}$ & $\begin{array}{l}\oplus \bigcirc \bigcirc \bigcirc \\
\text { very low }\end{array}$ \\
\hline Sexual satisfaction & $\begin{array}{l}\text { One study found higher levels } \\
\text { of satisfaction with the quality } \\
\text { of sexual relations with part- } \\
\text { ner (assessed as a subscale } \\
\text { of the Sexual Function for Car- } \\
\text { diac Patient Questionnaire) in } \\
\text { the intervention group in com- } \\
\text { parison to the control group. } \\
\text { Another study found no dif- } \\
\text { ference in sexual satisfaction } \\
\text { (assessed as a subscale of } \\
\text { the WSFQ) between interven- } \\
\text { tion and control groups at any } \\
\text { timepoint }\end{array}$ & $\begin{array}{l}207 \\
\text { (2 RCTs) }\end{array}$ & $\begin{array}{l}\oplus \bigcirc \bigcirc \bigcirc \\
\text { very low }\end{array}$ \\
\hline Relationship satisfaction & $\begin{array}{l}\text { One study found no difference } \\
\text { in relationship satisfaction } \\
\text { (assessed using Olson's En- } \\
\text { rich Marital Satisfaction ques- } \\
\text { tionnaire) between interven- } \\
\text { tion and control groups at any } \\
\text { timepoint }\end{array}$ & $\begin{array}{l}92 \\
(1 \mathrm{RCT})\end{array}$ & $\begin{array}{l}\oplus \bigcirc \bigcirc \bigcirc \\
\text { very low }\end{array}$ \\
\hline
\end{tabular}

Sexual counselling for sexual problems in patients with cardiovascular disease (Review)

Copyright @ 2016 The Cochrane Collaboration. Published by John Wiley \& Sons, Ltd. 


\begin{tabular}{|c|c|c|}
\hline Quality of life & $\begin{array}{l}\text { One study found no difference } 115 \\
\text { in quality of life (assessed us- (1 RCT) } \\
\text { ing the Ferrans and Powers } \\
\text { Quality of Life Index (QLI) Car- } \\
\text { diac Version III) between in- } \\
\text { tervention and control groups } \\
\text { at any timepoint }\end{array}$ & $\begin{array}{l}\oplus \bigcirc \bigcirc \bigcirc \\
\text { very low }\end{array}$ \\
\hline Anxiety & $\begin{array}{l}\text { One study found no difference } 207 \\
\text { in anxiety (assessed using (2 RCTs) } \\
\text { Speilberger's Anxiety Scale) } \\
\text { between intervention and con- } \\
\text { trol groups at any timepoint. } \\
\text { Another study found higher } \\
\text { levels of anxiety (assessed } \\
\text { using Speilberger's Anxiety } \\
\text { Scale) in the intervention } \\
\text { group in comparison to the } \\
\text { control group at one month } \\
\text { follow-up, but not at other } \\
\text { timepoints }\end{array}$ & $\begin{array}{l}\oplus \bigcirc \bigcirc \bigcirc \\
\text { very low } \\
1,2\end{array}$ \\
\hline $\begin{array}{l}\text { Resumption of sexual activ- } \\
\text { ity after a cardiac event (Re- } \\
\text { sumption of sex) }\end{array}$ & $\begin{array}{l}\text { All three studies reported the } 334 \\
\text { number of patients returning ( } 3 \text { RCTs) } \\
\text { to sexual activity following } \\
\text { myocardial infarction (MI) as } \\
\text { an outcome. One study found } \\
\text { some evidence of an effect } \\
\text { of sexual counselling on re- } \\
\text { ported rate of return to sexual } \\
\text { activity (yes/no) at } 4 \text { months } \\
\text { after completing the interven- } \\
\text { tion (relative risk (RR) } 1.71 \text {, } \\
95 \% \text { Cl } 1.26 \text { to } 2.32 ; 1 \text { trial, } \\
92 \text { participants, very low qual- } \\
\text { ity of evidence). Two studies } \\
\text { found no evidence of an effect } \\
\text { of sexual counselling on rate } \\
\text { of return to sexual activity at } \\
12 \text { weeks (RR } 1.01,95 \% \text { Cl } 0 \text {. } \\
94 \text { to } 1.09 ; 1 \text { trial, } 127 \text { partic- } \\
\text { ipants, very low quality of ev- } \\
\text { idence) and } 3 \text { months follow- } \\
\text { up (RR } 0.98,95 \% \text { Cl } 0.88 \text { to } \\
1.10 ; 1 \text { trial, } 115 \text { participants, } \\
\text { very low quality of evidence) }\end{array}$ & $\begin{array}{l}\oplus \bigcirc \bigcirc \bigcirc \\
\text { very low } 5,6\end{array}$ \\
\hline
\end{tabular}

Sexual counselling for sexual problems in patients with cardiovascular disease (Review) 
Satisfaction in how sexual is- No included studies reported (0 studies)

sues are addressed in cardiac satisfaction with how sexual

rehabilitation services (Satis- issues were addressed in car-

faction with health services) diac rehabilitation services

* The risk in the intervention group (and its $95 \% \mathrm{Cl}$ ) is based on the assumed risk in the comparison group and the relative effect of the intervention (and its $95 \% \mathrm{Cl}$ ).

Abbreviations: Cl: confidence interval; RR: risk ratio; OR: odds ratio; MI: myocardial infarction; RCT: randomised controlled trial; WSFQ: Watts Sexual Function Questionnaire.

\section{GRADE Working Group grades of evidence}

High quality: we are very confident that the true effect lies close to that of the estimate of the effect.

Moderate quality: we are moderately confident in the effect estimate: The true effect is likely to be close to the estimate of the effect, but there is a possibility that it is substantially different.

Low quality: our confidence in the effect estimate is limited: The true effect may be substantially different from the estimate of the effect.

Very low quality: we have very little confidence in the effect estimate: The true effect is likely to be substantially different from the estimate of effect

${ }^{1}$ Downgraded by one for risk of bias. One study was at unclear risk for sequence generation; 2 studies were at unclear risk for allocation concealment; 2 studies were at high risk for blinding of participants, incomplete outcome data, and other biases; and 2 studies were at unclear risk for blinding of outcome assessment.

${ }^{2}$ Downgraded by two for imprecision. The total sample size was less than 400 (this is a threshold rule-of-thumb value suggested by GRADE Working Group, which uses the usual alpha and beta, and an effect size of 0.2 SD, representing a small effect).

${ }^{3}$ Downgraded by one for risk of bias. This trial was at unclear risk for sequence generation, allocation concealment and blinding of outcome assessment; and at high risk for blinding of participants, incomplete outcome data, and other biases].

${ }^{4}$ Downgraded by one for risk of bias. This trials was at unclear risk for allocation concealment and blinding; and at high risk for blinding, incomplete outcome data, and other biases.

${ }^{5}$ Downgraded by two for risk of bias. Two studies were at unclear risk for sequence generation; three studies were at unclear risk for allocation concealment; three studies were at high risk for blinding of participants; incomplete outcome data, and other biases; and one study was at high risk and two studies were at unclear risk for blinding of outcome assessment.

${ }^{6}$ Downgraded by one for imprecision. The total number of events was less than 300 (a threshold rule-of-thumb value suggested by the Grade Working Group).

\section{B A C K G ROU N D}

\section{Description of the condition}

Sexual problems, such as erectile dysfunction among men and pain during intercourse among women, can occur frequently in relation to cardiac disease and its associated risk factors, medications, and psychological sequelae (Jaarsma 2010a; Jaarsma 2010b). Such problems are more prevalent among both men (Schumann 2010) and women (Kutmeç 2011) with cardiovascular disease than those without cardiovascular disease; the prevalence rates for men range from 20\% (Schumann 2010) to 70\% (Mulat 2010), and for women from $43 \%$ (Kriston 2010) to $87 \%$ (Schwarz 2008).

Reasons for the association between sexual problems and cardiovascular disease include physical vascular causes (Dong 2011), fear of sexual activity provoking cardiac symptoms or a cardiac event (Katz 2007), patient-partner relationship changes following a cardiac event (Dalteg 2011), and associations with psychological problems such as depression (Kriston 2010). Although there is evidence to suggest that some cardiac medications, including beta blockers and lipid-lowering medications, may have sexual side effects, more recent analyses concluded that cardiovascular medica- 
tions are uncommonly the true cause of sexual problems (Levine 2012).

Sexual dysfunction can impact negatively on quality of life, psychological well-being, and marital or partnership satisfaction (Traeen 2007; Günzler 2009). Social support and strong intimate relationships are important predictors of outcomes for people with chronic cardiovascular illness, and poor marital quality can predict patient mortality (Coyne 2001). Sexual problems also impact cardiac patients' partners, who rate sexual concerns as one of the most prevalent stressors related to their partner's condition $\left(\mathrm{O}^{\prime}\right.$ Farrell 2000).

Return to sexual activity after an acute cardiac event, or maintenance of a satisfactory sex life when living with chronic cardiovascular disease, can pose challenges for cardiovascular patients and their partners. It has been recommended that sexual problem assessment and counselling should form part of routine care for cardiovascular patients (Steinke 2013a). While those with cardiac disease view information about return to sexual activity as an important component of their general rehabilitation (Steinke 1998; Mosack 2009), health professionals have been reluctant to address sexual counselling in practice (Byrne 2010; Djurovic 2010; Ivarsson 2010; Jaarsma 2010c; Goossens 2011; D’Eath 2013), including staff in cardiac rehabilitation (Barnason 2011; Doherty 2011). Reasons for provider reluctance include a lack of confidence and education required to address these concerns adequately (Byrne 2010; Doherty 2011; Hoekstra 2012a).

\section{Description of the intervention}

"Counselling" refers to "systematic consultations in primary care for addressing emotional, psychological and social issues that influence a person's health and well-being" (WHO 2015). There is some evidence for the effectiveness of sexual counselling, psychoeducational, and psychological therapeutic approaches for sexual problems on general sexual function and satisfaction for general populations. A Cochrane review of psychosocial interventions for erectile dysfunction, which included 11 trials involving 398 men with erectile dysfunction, concluded that group psychotherapy was more likely than the control group (who received no treatment) to reduce the number of men with "persistence of erectile dysfunction” post-treatment (Melnik 2007). A systematic review in the area of cancer, which included eight trials, concluded that there was some evidence that psychoeducational interventions improved sexual function and reduced 'sexual bother' in men following prostatectomy for prostate cancer (Lassen 2013). Sexual counselling may also enhance the effectiveness of other treatments for sexual dysfunction. For example, sexual counselling improved selfadministration of pharmacological interventions for sexual dysfunction among people that received treatment for cancer (Miles 2007) and reduced levels of drop-out from drug therapy interventions that targeted erectile dysfunction among the general population (Melnik 2007).
Although most research focuses on sexual dysfunction in men, some research supports the effectiveness of psychoeducational interventions for sexual problems among women. For example, a brief three-session psychoeducational intervention significantly improved aspects of sexual response, mood, and quality of life in women with gynaecological cancer (Brotto 2008). A review of psychological interventions for couples coping with breast cancer concluded that they are effective in improving sexual functioning and sexual satisfaction among women (Brandão 2014).

The aims of sexual counselling (hereafter referred to as counselling) interventions for cardiac patients are to assess existing sexual problems, provide information on concerns, and support safe return to sexual activity after a cardiac event or procedure. Counselling interventions address specific psychological or interpersonal factors, sexual performance concerns, and issues related to medication and co-morbid conditions that may affect sexual functioning (Lue 2004). Non-sexual aspects of a relationship may also be addressed in a counselling intervention, such as the need for intimacy in the relationship (Steinke 2004). A range of different types of health professionals or other appropriately trained individuals may administer counselling interventions. These interventions may be delivered as separate, stand-alone interventions or as a component of more comprehensive rehabilitation interventions, such as in hospital cardiac rehabilitation following a cardiac event or procedure. Counselling interventions may involve a one-to-one exchange between a health professional and patient (in person or over the telephone) or may be delivered by a health professional to a group of cardiac patients. They may use one or a number of didactic and counselling approaches, including oral information or dialogue, visual information, written materials, audiovisual materials, and practical training. Counselling interventions may involve the cardiac patient alone or the cardiac patient with his or her partner or spouse. Interventions can be short-term, for example provision of brief information on return to sexual activity (Kushnir 1976; Fridlund 1991), or longer-term, for example providing cognitive behavioural therapy directed towards both psychological and physical aspects of sex and intimate relations (Klein 2007; Song 2011). Interventions may involve a single encounter or multiple encounters with a health professional.

Many issues related to both the health professional and the patient and their partner influence the delivery and effectiveness of counselling interventions. These can include gender and age differences of the health professional and the recipient, cultural and religious issues, and sexuality of the couple (Klein 2007; O'Donovan 2007; Hoga 2010; Goossens 2011). The nature and extent of the cardiac event itself may vary in complexity (Jaarsma 2010c), and more complex conditions require a more focused and specialised sexual counselling intervention (Ivarsson 2009; Ivarsson 2010). Health professionals' own beliefs about sexuality may influence the delivery of counselling interventions, for example health professionals may adhere to myths and biases regarding the need for counselling based on the age of the patient experiencing the cardiac event and 
their gender (Kazemi-Saleh 2008; Taylor 2011; Hoekstra 2012a; Hoekstra 2012b). Apart from the individual health professional, organisational structures related to financial resources, availability of staff, time restrictions, and availability of private spaces can impact on the delivery and organisation of counselling interventions (Song 2011; Steinke 2012).

\section{How the intervention might work}

Counselling interventions are likely to work by providing useful information, which may reduce anxiety related to sexual problems and fears about resuming sexual activity after a cardiac event. They may also increase confidence in sexual abilities and potential, improve patient-partner communication around changes to sexual activity required following a cardiac event, provide practical guidance, and teach skills to support couples in returning to sex. In such interventions, health professionals may assess any risk associated with sexual activity and develop an individualised plan to guide safe resumption of sexual activity following a cardiac event or procedure (Gamel 1993; Levine 2012). Such information is likely to alleviate fears associated with return to sexual activity and provide patients and partners with greater confidence in their ability to assess if, and when, it is right for them to return to sexual activity. Counselling interventions aim to provide correct information and dispel myths about how cardiac disease impacts on sexual activity. By giving cardiac patients the opportunity to express their sexual concerns, interventions in this area are likely to 'normalise' these concerns and reassure patients and their partners that sexual problems are common after a cardiac event and can be addressed. Counselling interventions may also provide practical guidance to patients about how to return to sexual activity (Mosack 2009). Such guidance may include aspects such as ideal timing (when the patient is not tired) and setting (comfortable and familiar), and warn against things that may increase risks associated with sexual activity, for example sex should generally be avoided following a heavy meal (Levine 2012).

The effectiveness of interventions can be evaluated by assessment of outcomes that reflect the ways in which the intervention is likely to work. These include changes in sexual activity levels and resumption of sexual activity following a cardiac event or procedure, sexual knowledge, sexual function and satisfaction, and quality of life (Bertie 1992; Klein 2007; Song 2011; Steinke 2012).

\section{Why it is important to do this review}

Worldwide, cardiovascular disease is a leading cause of morbidity and mortality. However, survival rates are increasing which is resulting in an increasing number of people living in the community with some form of cardiovascular disease (WHO 2011). Counselling for patients and their partners or spouses has been recommended as an important component of cardiac rehabilita- tion (Levine 2012; Steinke 2013a). There is ample literature to indicate that counselling of cardiac patients is infrequently provided in practice (Steinke 1998; Goossens 2011; Steinke 2011a). However, when asked, cardiac patients (Byrne 2013) and their partners (O’ Farrell 2000; Fisher 2005; Agren 2009) generally report that this is something they would appreciate. While health professionals report responsibility for, and some knowledge of, counselling provision (Steinke 2011b), there is a lack of follow-through in implementation of counselling interventions in daily practice (Pouraboli 2010; Y ld z 2012). Published trials have examined the effectiveness of counselling interventions, yet there is currently no systematic review of these studies. A well-conducted systematic review is needed to inform health professionals, patients and their partners, and policy makers about the effectiveness of such interventions. In addition, an evaluation of interventions may provide insights into which strategies might be most or least effective for cardiac patients, as well as which interventions may be most amenable to use in busy practice settings by health professionals.

\section{O B J E C T I V E S}

To evaluate the effectiveness of sexual counselling interventions (in comparison to usual care) on sexuality-related outcomes in patients with cardiovascular disease and their partners.

\section{METHODS}

\section{Criteria for considering studies for this review}

\section{Types of studies}

Randomised controlled trials (RCTs) and quasi-RCTs (including individual and cluster RCTs) were eligible for inclusion. We included studies that compared any form of sexual counselling with usual care.

\section{Types of participants}

Adults (aged 18 years or more) with cardiac disease including those who experienced a myocardial infarction (MI), a revascularisation procedure (coronary artery bypass grafting (CABG) or percutaneous transluminal coronary angioplasty), those with angina or angiographically-defined coronary heart disease, heart failure, and congenital heart disease. We also included participants with heart transplants or implanted with either cardiac resynchronisation therapy or implantable defibrillators. We included the partners of these patients, when they were included in the study. 


\section{Types of interventions}

We considered all interventions designed to address and counsel cardiac patients in relation to sexual problems that may have arisen as a result of their cardiac condition.

For the purpose of this Cochrane review we used the following operational definitions.

A sexual counselling intervention is any intervention delivered by a health professional or appropriately trained individual (for example, a sex therapist) with the aim of providing cardiac patients with information on sexual concerns and safe return to sexual activity after a cardiac event or procedure, as well as assessment, support, and specific advice related to psychosexual and sexual problems related directly to their cardiac condition. Sexual counselling interventions may be delivered as a component of hospital cardiac rehabilitation following a cardiac event or procedure. Sexual counselling interventions may involve a one-to-one exchange between a health professional and patient (in person or over the telephone) or may be delivered by a health professional to a group of cardiac patients. Sexual counselling interventions may use one or more didactic approaches, including oral information or dialogue, visual information, written materials, audiovisual materials, and practical training. Sexual counselling interventions may involve the cardiac patient alone or the cardiac patient with his or her partner or spouse. Sexual counselling interventions can be shortterm (for example, brief provision of information and counselling in the acute care setting) or longer-term (for example, ongoing counselling in the office setting on repeat patient visits) and may involve a single encounter with a health professional or multiple encounters.

We only considered trials where the comparison group was usual care or no sexual counselling intervention, and reported follow-up for at least three months post-intervention. 'Usual care' consisted of standard cardiac care, without the addition of sexual counselling as described above.

\section{Types of outcome measures}

We included outcome measures from both patients with cardiac disease and their partners, where available.

\section{Primary outcomes}

\section{Participant}

- Sexual function or sexual dysfunction, using validated instruments including:

- Index of Erectile Function-5 (IIEF-5) (Rosen 1997);

- Brief Male Sexual Function Inventory (BMSFI)

(Mykletun 2006);

- Female Sexual Function Index (FSFI) (Rosen 2000);

- Brief Index of Sexual Functioning for Women (BISF-

W) (Taylor 1994);
- Derogatis Interview for Sexual Functioning (DISF) and Derogatis Interview for Sexual Functioning - Self-Report (DISF-SR) (Derogatis 1997);

- Changes in Sexual Functioning Questionnaire (CSFQ) (Clayton 1997) and Changes in Sexual FunctioningShort Form (CSFQ-SF) (Keller 2006);

- Arizona Sexual Experience Scale (ASEX) (McGahuey 2000);

- Sexual Function Questionnaire (SFQ) (Syrjala 2000);

- Sexual Function Questionnaire (Quirk 2002);

- European Male Ageing Study Sexual Function

Questionnaire (EMAS-SFQ) (O’Connor 2008).

- Sexual satisfaction, using validated instruments including:

- Sexual Self-Perception and Adjustment Questionnaire (SSPAQ) (Steinke 2013b);

- Sexual Satisfaction Scale for Women (SSS-W)

(Meston 2005).

\section{Partner}

- Sexual satisfaction, using validated instruments including: - SSPAQ (Steinke 2013b).

\section{Secondary outcomes}

\section{Patient}

- Marital or relationship satisfaction, using validated instruments including:

- ENRICH Marital Satisfaction Scale (Fowers 1993).

- Quality of life, using validated instruments including:

- Ferrans and Powers Quality of Life Index (QLI) -

Cardiac Version III (Ferrans 1985);

- 36-item Short Form Health Survey (SF-36) (Ware

1992) or the 12-item Short Form Health Survey (SF-12) (Ware 1995).

- Psychological well-being (including anxiety and depression), using validated instruments including:

- Speilberger's State-Trait Anxiety Inventory (Speilberger 1983).

- Satisfaction in how sexual issues are addressed in cardiac rehabilitation services.

- Resumption of sexual activity after a cardiac event: (a) presence or absence of sexual activity; (b) frequency of sexual activity; and (c) time taken to resume sexual activity after cardiac event or procedure, using validated instruments including:

- Return to Sexual Activity Inventory (Steinke 2004).

- Knowledge about sex after an MI, using a validated instrument such as:

- 25-item Sex After MI Knowledge Test (Steinke 2004). We did not include this outcome in the protocol for this review, 
Byrne 2014, but we later identified and included it in the review as a potentially useful outcome to consider.

\section{Partner}

- Satisfaction in how sexual issues are addressed in cardiac rehabilitation services.

- Marital or relationship satisfaction, using validated instruments including:

- ENRICH Marital Satisfaction Scale (Fowers 1993).

- Quality of life or psychological well-being, using validated instruments including:

- SF-36 (Ware 1992) or the SF-12 (Ware 1995).

\section{'Summary of findings' table}

We only included outcomes stated in the Cochrane protocol, Byrne 2014, in the 'Summary of findings' table of this Cochrane review. We have listed these outcomes below.

- Sexual function.

- Sexual satisfaction.

- Relationship satisfaction.

- Quality of life.

- Psychological well-being/anxiety.

- Resumption of sexual activity after a cardiac event.

- Satisfaction in how sexual issues are addressed in cardiac rehabilitation services.

\section{Search methods for identification of studies}

\section{Electronic searches}

The Cochrane Heart Group Trials Search Co-ordinator searched the following databases up to 2 March 2015 (except CENTRAL) without restrictions on language: CENTRAL (Issue 1 of 12, 2015; the Cochrane Library) (searched 21 March 2015); MEDLINE (OVID) (1946 to Feb week 4 2015); EMBASE (OVID) (1980 to 2015 week 09); CINAHL Plus with Full Text (EBSCO) (1937 to 02 March 2015); PsycINFO (OVID) (1806 to Feb week 4 2015); Conference Proceedings Citation Index - Science (CPCIS) on Web of Science (Thomson Reuters) (1990 to 27 February 2015). We searched Clinicaltrials.gov (www.clinicaltrials.gov) and the World Health Organization International Clinical Trials Registry Platform (WHO ICTRP) (http://apps.who.int/trialsearch/) up to 03 February 2016 and used the terms 'counselling' and 'sexual dysfunction'.

In the protocol, Byrne 2014, we proposed that we would "search reference lists of eligible papers and reviews" and "contact the principal investigators of identified studies to ascertain if they are aware of any other relevant published or unpublished studies in the area". We did not conduct these additional searches of other resources as we, internationally experienced people in this field, considered it highly likely that we captured all ongoing intervention research activity via the core search strategies.

We applied the Cochrane sensitivity maximising RCT filter (Lefebvre 2011) to MEDLINE and adaptations of it to the other databases as applicable. The search strategies and search terms for all the databases are in Appendix 1.

\section{Searching other resources}

We did not conduct any formal additional searches of resources. As outlined above, we considered it highly likely that we captured all ongoing intervention research activity via the core search strategies.

\section{Data collection and analysis}

We used the Cochrane Handbook for Systematic Reviews of Interventions to inform the Methods (Higgins 2011).

\section{Selection of studies}

One review author (MB) imported citations into a reference management software package (EndNote), and removed duplicates. $\mathrm{MB}$ then imported citations into the Covidence online systematic review management system (www.covidence.org/). Using Covidence, two review authors (MB and SD) independently screened titles and abstracts for potentially eligible studies. We resolved any discrepancies by consensus.

We retrieved full-text publications of potentially eligible studies. Two review authors (MB and SD) independently determined study eligibility using a standardised inclusion form. We resolved any disagreements about study eligibility by discussion and, if necessary, we asked a third review author (DD) to arbitrate.

In the 'Characteristics of excluded studies' table we have listed all potentially eligible papers that we excluded from the review at the full-text stage, along with the reasons for exclusion.

We reported the screening and selection process in an adapted PRISMA flow chart. While we identified no ongoing studies in this Cochrane review, in future updates we will provide citation details and any available information about ongoing studies.

\section{Data extraction and management}

For included studies, two review authors (MB and SD) independently extracted study characteristics and outcome data using a standardised data collection form we created for this Cochrane review.

We extracted the following data from each included study.

- Study details: author, year, research question, or study aim; country where the research was carried out; recruitment source (e.g. patients attending hospital cardiac rehabilitation); inclusion and exclusion criteria; study design (RCT; individual or cluster 
RCT, and single- or multi-centre); length of follow-up; description of usual care.

- Intervention details: setting of intervention (hospital, general practice); delivered to individuals or groups; targeting patients only, or patient and partner dyads; degree of training of the person who provided the intervention; topics covered in the intervention; number of sessions in intervention; overall duration of intervention; timing of delivery of intervention in relation to cardiac history; mode of intervention (e.g. written information, DVD, lecture or talk, individual counselling).

- Participant characteristics: primary cardiac diagnosis; age; sex; socioeconomic status; ethnicity; reported co-morbidities.

- Primary and secondary outcomes.

- Numbers of participants randomised and assessed at specified follow-up points.

- Adherence to intervention and rate of attrition.

We resolved any discrepancies in data extraction by consensus. One review author (MB) transferred the extracted data into Review Manager (RevMan) (RevMan 2014) and a second review author (SD) spot-checked the data for accuracy.

\section{Assessment of risk of bias in included studies}

Two review authors (MB and SD) independently assessed the risk of bias in included studies using the Cochrane 'Risk of bias' assessment tool (Higgins 2011). The'Risk of bias' assessment comprised a judgement and a support for the judgement for each entry in a 'Risk of bias' table, where each entry addressed a specific feature of the study. The judgement for each entry involved assessment of the risk of bias as either 'low risk', 'high risk, or 'unclear risk', and the last category indicated either lack of information or uncertainty over the potential for bias.

We included the following 'Risk of bias' items.

- Random sequence generation (checking for possible selection bias).

- Allocation concealment (checking for possible selection bias).

- Blinding of participants and personnel (checking for possible performance bias).

- Blinding of outcome assessment (checking for possible detection bias).

- Incomplete outcome data (checking for possible attrition bias through withdrawals, people lost to follow-up, protocol deviations).

- Selective reporting (checking for reporting bias).

- Other bias (checking for other potential sources of bias not covered in the categories above).

Three review authors $(\mathrm{MB}, \mathrm{SD}$, and $\mathrm{DD})$ resolved any discrepancies regarding 'Risk of bias' assessments by consensus.

\section{Measures of treatment effect}

For dichotomous data, we presented the results as summary risk ratios (RRs) with 95\% confidence intervals (CIs) where possible. For continuous data, we used the mean difference value with $95 \%$ CI for outcomes. In future updates, we will use the standardised mean difference with $95 \% \mathrm{CI}$ to combine data that measured the same outcome but used different scales.

\section{Unit of analysis issues}

We did not identify any cluster RCTs in our literature searches. In future updates of this Cochrane review, if we identify any cluster RCTs we will include them along with individually RCTs. We will adjust their sample sizes using the methods described in the Handbook for Systematic Reviews of Interventions (Higgins 2011) and using an estimate of the intracluster correlation coefficient (ICC) derived from the trial (if possible), from a similar trial, or from a study of a similar population. If we use ICCs from other sources we will report this and conduct sensitivity analyses to investigate the effect of variation in the ICC. If we identify cluster RCTs and individually RCTs, we will synthesise the relevant information. We will consider it reasonable to combine the results from both where there is little heterogeneity between the study designs and where we consider that there is unlikely to be an interaction between the effect of the intervention and the choice of randomisation unit. We will acknowledge heterogeneity in the unit of randomisation and perform a sensitivity analysis to investigate the effects of this heterogeneity on the review findings.

\section{Dealing with missing data}

We noted the levels of attrition in the included studies. However, we were unable to perform any data analysis in this review. If possible, we will perform analyses on an intention-to-treat basis for all outcomes in future updates of this Cochrane review.

\section{Assessment of heterogeneity}

We were unable to pool data, and therefore we did not need to consider statistical heterogeneity.

In future updates of this review, if we are able to pool data, we will assess statistical heterogeneity in each meta-analysis using the $\mathrm{Tau}^{2}$ (tau-squared) statistic, $\mathrm{I}^{2}$ statistic, and $\mathrm{Chi}^{2}$ test. We will regard heterogeneity as substantial if the $\mathrm{I}^{2}$ statistic value is high (above 30\%); and either there is inconsistency between trials in the direction or magnitude of effects (judged visually), or a low (less than 0.10$) \mathrm{P}$ value in the $\mathrm{Chi}^{2}$ test for heterogeneity or the estimate of between-study heterogeneity $\left(\mathrm{Tau}^{2}\right)$ is above zero.

\section{Assessment of reporting biases}

As fewer than 10 studies met the inclusion criteria of this Cochrane review, we did not investigate publication bias using funnel plots. 
In future updates of this review, if we include 10 or more studies in a meta-analysis, we will investigate publication bias using funnel plots by assessment of funnel plot asymmetry visually and by use of formal tests for funnel plot asymmetry. For continuous outcomes we will use the test proposed by Egger 1997, and for dichotomous outcomes we will use the test proposed by Harbord 2006. If we detect asymmetry in any of these tests, or it is suggested by a visual assessment, we will perform exploratory analyses to investigate it.

\section{Data synthesis}

We did not conduct any data synthesis.

We produced a narrative 'Summary of findings' table (Higgins 2011) using the GRADEpro Guideline Development Tool (GDT) (www.gradepro.org). We summarised the quality of evidence and the strength of recommendations using the Grading of Recommendations Assessment, Development and Evaluation (GRADE) approach for each of the seven outcomes pre-specified in our Cochrane protocol (Byrne 2014).

In future updates of this review, if meta-analysis of results is possible (as diversity is likely in the types and content of interventions included in the trials), we will use a random-effects model metaanalysis to produce an overall summary of the average treatment effect across all included trials. For each reported outcome, we will present the results of the random-effects model analyses as the average treatment effect with its $95 \% \mathrm{CI}$ and the estimates of the $\mathrm{Tau}^{2}$ and the $\mathrm{I}^{2}$ statistic.

\section{Subgroup analysis and investigation of heterogeneity}

We were unable to conduct any subgroup analysis as we did not pool findings from individual studies. In future updates of this review, if we include a sufficient number of studies in the metaanalyses (10 trials should be available for each characteristic modelled), we will perform subgroup analyses for the following.

- Delivery mode of intervention: intervention delivered to group versus delivered to individual.

- Delivery mode of intervention: 'face to face' versus 'distance delivery'.
- Target of intervention: intervention delivered to individuals (cardiovascular patients only) versus delivered to dyads or couples (cardiovascular patients plus their partners).

- Gender of intervention participant: male versus female.

We will assess subgroup differences by the interaction tests available in RevMan (RevMan 2014). We will report the results of subgroup analyses and quote the $\mathrm{Chi}^{2}$ test and $\mathrm{P}$ value, and the $\mathrm{I}^{2}$ statistic value of the interaction test.

\section{Sensitivity analysis}

We did not perform a sensitivity analysis as we were unable to pool data from the individual included studies. In future updates of this Cochrane review, we will perform a sensitivity analysis by limiting analyses to studies at low risk of bias. We will do this by exclusion of studies at 'high' or 'unclear' risk of bias for sequence generation, allocation concealment, and incomplete outcome data.

\section{R E S U L T S}

\section{Description of studies}

See the 'Characteristics of included studies' and 'Characteristics of excluded studies' tables.

\section{Results of the search}

We identified 6958 records through our electronic database searches and 11 through clinical trial register searches. After deduplication, we screened 5549 abstracts for inclusion and excluded 5524 records. We retrieved 25 articles for full-text review and assessed them for eligibility; we then excluded 22 studies (see the 'Characteristics of excluded studies' table). In total we included three papers (see the 'Characteristics of included studies' table) that reported three separate studies (see Figure 1 for the PRISMA flowchart). 
Figure I. Study PRISMA flow diagram.

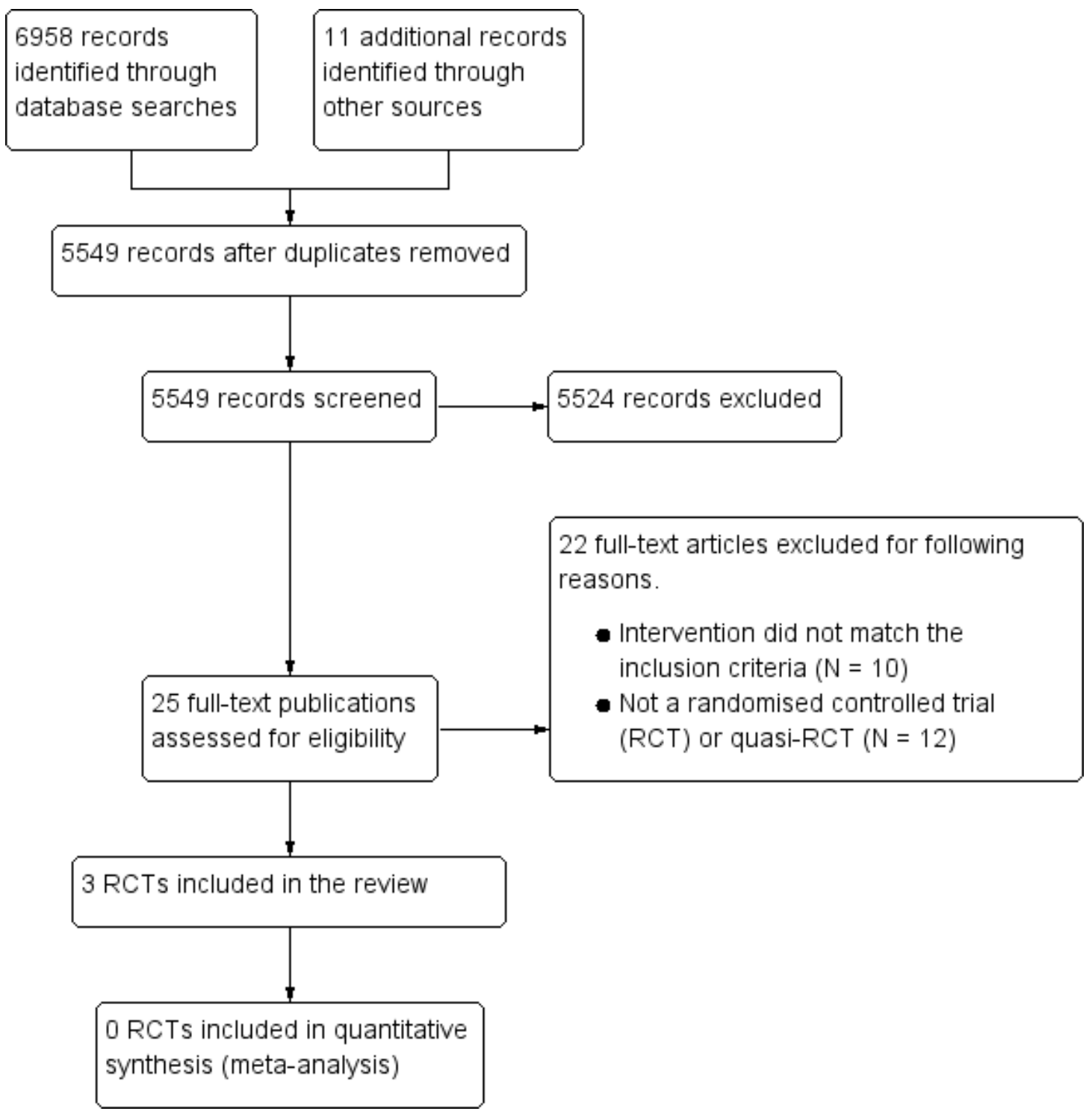

\section{Included studies}

We described the interventions according to what the study authors wrote in the papers (see the 'Characteristics of included studies' table). We did not contact the study authors for further information regarding intervention content. The included studies were undertaken in the USA (Froelicher 1994; Steinke 2004) and Israel (Klein 2007). All studies included participants who had been admitted to hospital with myocardial infarction (MI); Klein 2007 included participants who had undergone coronary artery bypass grafting $(\mathrm{CABG})$, in addition to participants with MI. The interventions in the three studies differed substantially, and therefore meaningful pooling of results from individual studies was not possible.

The intervention in Froelicher $1994(\mathrm{~N}=174)$ was usual care plus an exercise programme plus an education-counselling cardiac rehabilitation programme that contained a component that focused on resumption of sexual activity following a MI. This study had two comparison groups: one received usual care only, and the other received usual care plus an exercise programme. As we were 
interested in the added benefit of the sexual education-counselling intervention, we treated the group that received usual care plus an exercise programme as the control group, and usual care plus an exercise programme plus an education-counselling cardiac rehabilitation programme with the component focused on sexual activity as the intervention group.

Klein $2007(\mathrm{~N}=92)$ used a sexual counselling intervention that consisted of three meetings (total five hours) between a sexual therapist(s) and the participant (with the option of including their sexual partner). The counselling sessions involved education around issues related to sexual activity in general and after MI and CABG in particular; instructions, assignments, and sensate focusing exercises; discussion of experience with the exercises, cognitive behavioural techniques, and additional medical checks and medication prescription where necessary. Participants in the control group underwent the regular cardiac rehabilitation programme.

The intervention in Steinke $2004(\mathrm{~N}=115)$ was a 15 -minute educational video plus written material developed by clinical experts and distributed to participants in the intervention group before they left hospital following a MI. Participants in the control group received the written material only (usual care). The video contained content on the effect of the heart attack on sexuality and sexual function, communicating with the partner, the impact of cardiac risk factors on sexual function, specific suggestions on when and how to resume sexual activity, and the effects of various medications on sexual function.

\section{Excluded studies}

Of the 5539 unique citations detected, we excluded 5514 after title and abstract screening. We excluded a further 22 studies after we screened the full-text article and listed the reasons in the' Characteristics of excluded studies' table.

\section{Risk of bias in included studies}

We have summarised the 'Risk of bias' results in Figure 2 ('Risk of bias' summary: review authors' judgments about each 'Risk of bias' item for each included study) and Figure 3 ('Risk of bias' graph: review authors' judgments about each 'Risk of bias' item presented as percentages across all included studies). Incomplete reporting was an obstacle to the assessment of bias or quality of all three included studies. 
Figure 2. 'Risk of bias' summary: review authors' judgements about each 'Risk of bias' item for each included study.

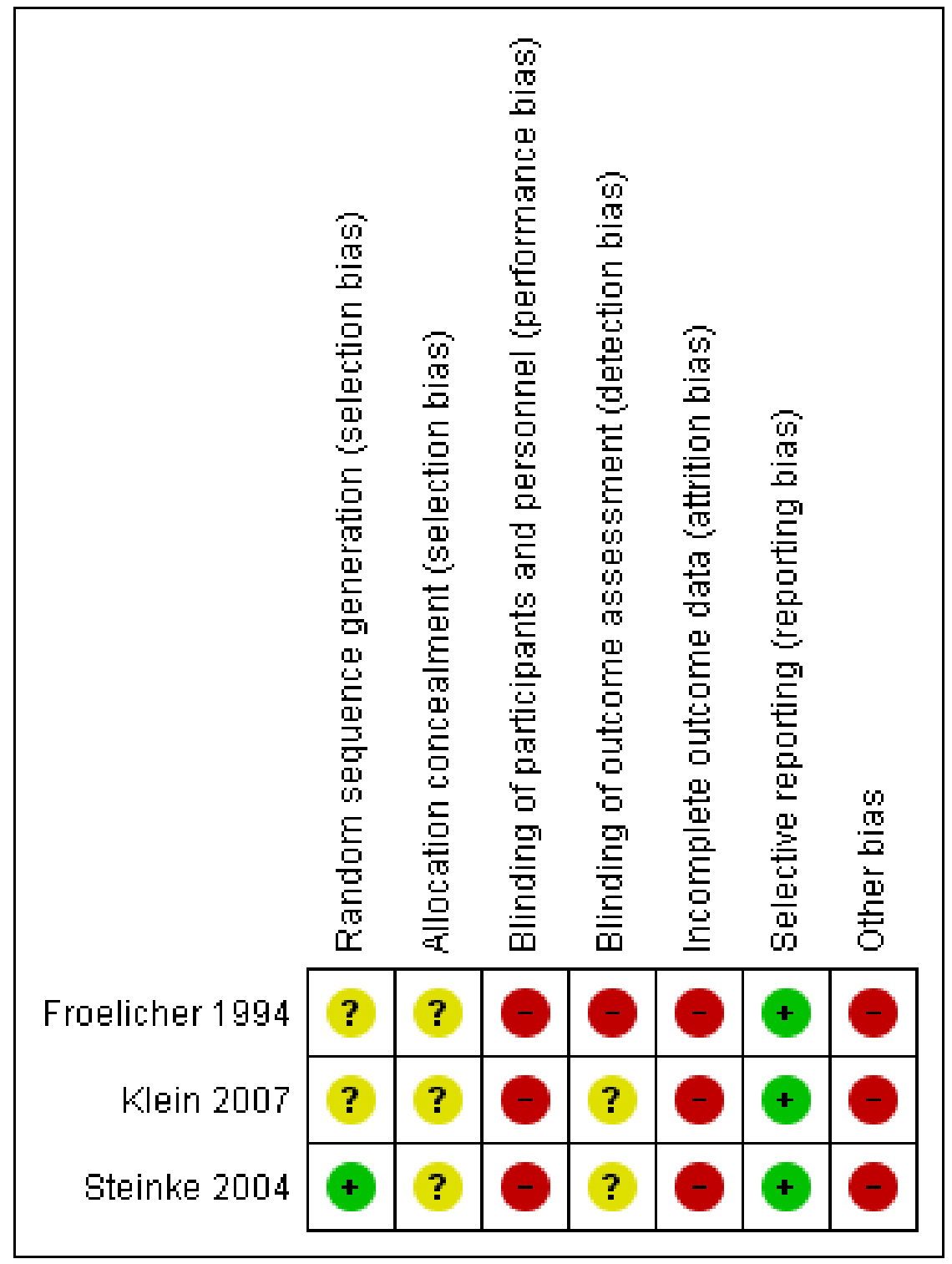


Figure 3. 'Risk of bias' graph: review authors' judgements about each 'Risk of bias' item presented as percentages across all included studies.

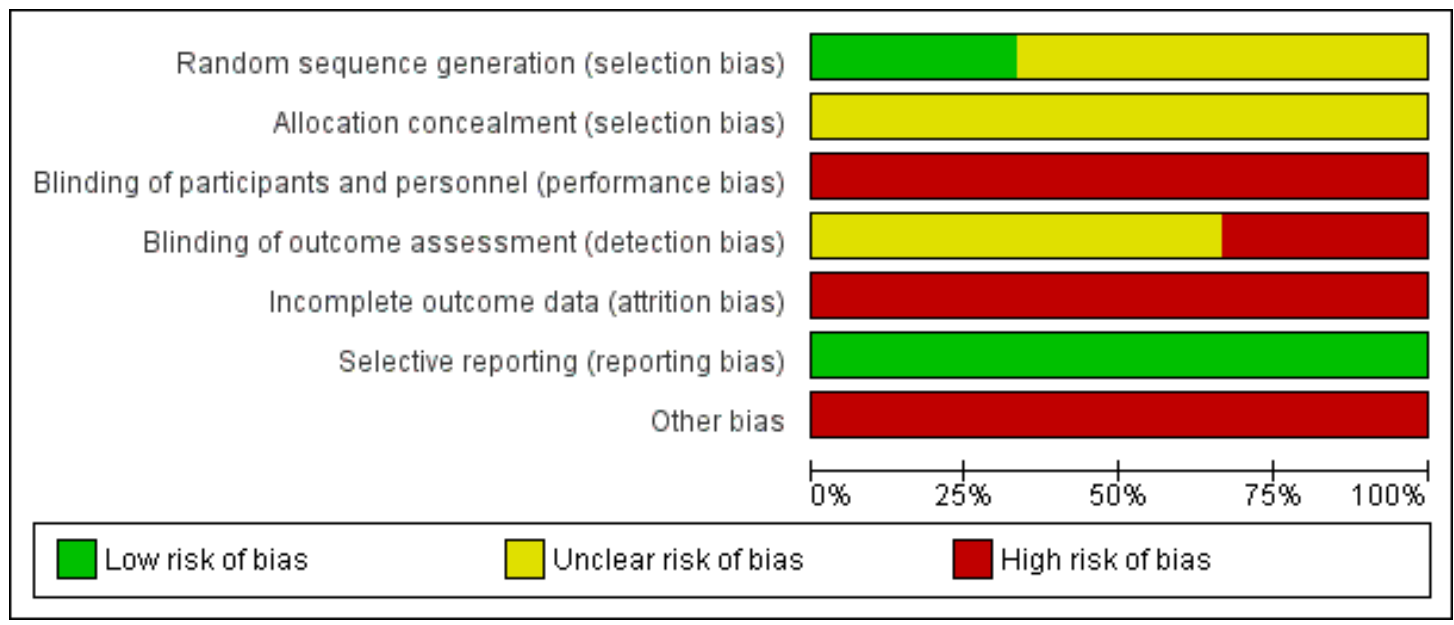

\section{Allocation}

We judged only one study as at low risk of bias in relation to adequate random sequence generation (Steinke 2004). The other two studies did not provide any details of this process (Froelicher 1994; Klein 2007), so we judged these as 'unclear'. We deemed all three included studies at unclear risk regarding allocation concealment, as the studies did not provide any details on the process.

\section{Blinding}

We judged all three included studies as at high risk of performance bias, as blinding of participants to their group allocation is not possible in this type of research.

Froelicher 1994 was at high risk of detection bias as research nurses were involved in both intervention delivery as well as data collection. We judged detection bias as unclear for Steinke 2004 and Klein 2007, as these studies provided limited details regarding whether those collecting the outcome data were blinded to group assignment.

\section{Incomplete outcome data}

We deemed all three included studies at high risk of attrition bias as they reported greater than $20 \%$ missing data for the main analysis. In Froelicher 1994, the attrition rate for the intervention and control groups was $25 \%(44 / 174)$ at 24 weeks follow-up. Data were unavailable to determine overall attrition rates at 12 weeks follow-up.

In Klein 2007, the attrition rate was $42 \%$ (38/92) at four months follow-up for sexual satisfaction. Data were unavailable to determine attrition rates for other outcomes at four months follow-up or any attrition rates at one month follow-up.

In Steinke 2004, the attrition rate was 34\% (39/115) at one month follow-up, $37 \%(43 / 115)$ at three months follow-up, and $40 \%$ $(46 / 115)$ at five months follow-up.

\section{Selective reporting}

There were an insufficient number of included studies to test for publication bias using a funnel plot. However, we minimised reporting bias by conducting a comprehensive search for studies that met the eligibility criteria. Furthermore, included studies did not provide strong evidence that sexual counselling can improve sexual outcomes for patients, and information from experts did not suggest that there were relevant unpublished studies.

Selective reporting of outcomes cannot be excluded as no study protocols were available. However all outcomes mentioned in the methods were reported in the results sections.

\section{Other potential sources of bias}


All three included studies were at high risk regarding additional sources of bias as they used self-reported measures for their outcomes. Such measures are subject to reporting bias, whereby participants are at risk of selectively revealing or suppressing information.

The intervention in Froelicher 1994 was conducted between 1977 and 1979, but the paper included in this review was published in 1994. Perhaps as a result, the level of detail reported of the intervention content lacked specificity. In addition, the time difference between study conduct and study reporting may cause additional problems in interpretation and modern relevance of the study's findings. The study authors noted that "These data were collected between 1977 and 1979. Thus temporal changes that have occurred since then, such as treatment alternatives, medications and other practice changes, may have different effects today".

In Klein 2007, the study authors reported that differences observed at baseline between intervention and control groups may have biased the results. They stated: "The significant differences observed between the treatment and control groups with regard to the proportions with CABG and previous $\mathrm{MI}$ is a potentially important limitation". More participants reported a previous CABG in the intervention group $(21 / 47,44.7 \%)$ compared to the control group (12/45, 26.7\%). More participants in the control group reported a previous $\mathrm{MI}(37 / 45,82.2 \%)$ than in the intervention group $(28 / 47,60.0 \%)$. They suggested that future studies should stratify for these pre-existing characteristics. The study authors reported that their small sample size may be a limitation. They stated that "the sample size does not provide enough power to detect smaller effects. In fact, several outcome measures showed a promising trend, but the limited power of our sample did not allow the detection of significant effects".

In Steinke 2004, there was a potential threat to external validity as the manager of cardiac rehabilitation identified and selected participants for inclusion. Generalisability of study findings may be limited, as the study authors stated that "participants in the study were primarily married, white and educated, thereby limiting the generalisability of the findings". Small sample size was another potential source of bias. The study authors stated: "Through power analysis it was anticipated that 45 participants were needed for each group". However, this sample size was not achieved at any of the follow-up time

points. Data were available for 76 participants in total at one month follow-up, for 72 participants as three months follow-up, and 69 participants at five months follow-up. There was also a greater attrition rate reported for the intervention group in comparison to the control group ( $45 \%$ versus $27 \%$ ).

\section{Effects of interventions}

See: Summary of findings for the main comparison Sexual counselling compared with usual care for patients with cardiovascular disease

\section{Primary outcomes - patients}

\section{Sexual function}

Two included studies reported sexual function as an outcome ( Steinke 2004; Klein 2007).

Klein 2007 assessed sexual function at baseline (entry to the intervention), one month, and four months follow-up. Sexual function was measured using the Sexual Function for Cardiac Patient Questionnaire, which is a compilation of items from several sources, including the International Index of Erectile Dysfunction. It includes 12 items, namely: fear to have sex; desire; confidence in maintaining an erection; satisfactory sexual relationship; sexual pleasure; frequency of erection; erection solid enough for penetration; satisfaction in frequency of sex; premature ejaculation; achieving orgasm; frequency of satisfaction in sexual intercourse; and health problems during sexual relations. The study authors developed this tool.

The study authors reported mean scores for each of the 12 items at each of the three timepoints for intervention and control groups. Scores for each item ranged from one (not at all/never) to five (a large extent/always). They conducted a repeated measured analysis of variance (ANOVA) test to compare intervention and control groups in magnitude of change in mean score across the three timepoints for each scale item. They reported only the results of statistical analysis for the items for which they found statistically significant differences. They reported differences in favour of the intervention in confidence in maintaining an erection $(\mathrm{F}(2,72)$ $=7.32, \mathrm{P}<0.001)$, satisfactory sexual relationships $(\mathrm{F}(2,53)=$ 4.23, $\mathrm{P}<0.02)$, frequency of erection $(\mathrm{F}(2,53)=4.23, \mathrm{P}<0.02)$, joy of sex $(\mathrm{F}(2,58)=3.35, \mathrm{P}<0.04)$ and levels of sexual desire $(\mathrm{F}(2,58)=3.16, \mathrm{P}<0.04)$. There were no differences reported on the remaining indices on this questionnaire.

Steinke 2004 assessed sexual function at pretest and at 1, 3, and 5 months follow-up. Sexual function was measured using the Watts Sexual Function Questionnaire (WSFQ), which is a 17-item scale with 4 subscales, i.e. sexual desire, arousal, orgasm, and satisfaction. Sexual function was calculated as a total score of all four WSFG items. Raw scores for sexual function were not reported. The study authors reported the results of a repeated measures analysis of covariance (ANCOVA) of changes in means over the three timepoints. In these analyses they included the covariates of age, gender, level of education, return to sexual activity, and prior value of the variable being considered. In describing their results from this analysis in relation to sexual function, the study authors stated: "There were no significant differences between the two groups [for sexual function] at any of the timepoints".

\section{Sexual satisfaction}

Two included studies reported sexual satisfaction as an outcome (Steinke 2004; Klein 2007). 
In Klein 2007, sexual satisfaction was assessed by one of the 12 items of the Sexual Function for Cardiac Patient Questionnaire (described above) 'satisfactory sexual relationship (or satisfaction with the quality of sexual relationships with partner)'. Scores for this item ranged from one (not at all/never) to five (a large extent/ always). The study authors reported mean indices (where a higher score indicates higher levels of satisfaction) for intervention group at time 1 , time 2 , and time 3 as $3.75,4.62$, and 4.37, and for the control group at time 1, time 2, and time 3 as 3.75, 3.71, and 3.63. They reported the findings of a repeated measured ANOVA test to compare intervention and control groups in magnitude of change in mean 'satisfactory sexual relationships' score across the three timepoints, and stated that "differences were reported in favour of the intervention $[\mathrm{F}(2,53)=4.23, \mathrm{P}<0.02]$ ”.

Steinke 2004 assessed sexual satisfaction and reported scores from one of the four subscales of the Watts Sexual Function Questionnaire (WSFQ) (described above). Raw scores for sexual satisfaction were not reported. The study authors reported results of a repeated measures ANCOVA of changes in means over the three timepoints, and stated: "There were no significant differences between the two groups [for sexual satisfaction] at any of the timepoints".

\section{Primary outcomes - partners}

None of the included studies reported any outcomes from partners.

\section{Secondary outcomes}

\section{Marital or relationship satisfaction}

One study reported marital satisfaction as an outcome (Klein 2007).

Klein 2007 assessed marital satisfaction at baseline (entry to the intervention), one month, and four months follow-up using Olson's Enrich Marital Satisfaction questionnaire. No scores were reported, but the study authors stated: "No treatment effects were observed on marital satisfaction as measured in three points in time".

\section{Quality of life}

One study reported quality of life as an outcome (Steinke 2004). Steinke 2004 assessed quality of life at pretest and at 1, 3, and 5 months follow-up using the Ferrans and Powers Quality of Life Index (QLI) - Cardiac Version III. Raw scores for quality of life were not reported. The study authors reported results of a repeated measures ANCOVA of changes in means over the three timepoints, and stated: "There were no significant differences between the two groups [for quality of life] at any of the timepoints".

\section{Psychological well-being}

Two studies reported psychological outcomes, and in both cases assessed anxiety using the Speilberger's State Trait Anxiety Inventory (Steinke 2004; Klein 2007).

Anxiety was assessed in Klein 2007 at baseline (entry to the intervention), and at one and four months follow-up. No scores were reported but the study authors stated: "No treatment effects were observed on state anxiety as measured in three points in time".

Anxiety was assessed in Steinke 2004 at baseline and at 1, 3, and 5 months follow-up. No scores were reported, but based on results of a repeated measures ANCOVA of changes in means over the three timepoints, they stated: "The experimental group had significantly greater anxiety at one month post $\mathrm{MI}[\mathrm{F}(2,75)=2.78, \mathrm{P}<0.05]$. The study authors reported: "There were no significant differences between the two groups [for anxiety] at any other timepoints".

\section{Satisfaction in how sexual issues are addressed in cardiac rehabilitation services}

No study reported this outcome.

\section{Resumption of sexual activity after a cardiac event}

Three included studies reported resumption of sexual activity after a cardiac event as an outcome (Froelicher 1994; Steinke 2004; Klein 2007).

All three studies reported the number of patients that returned to sexual activity following MI as an outcome.

While Klein 2007 reported significant differences between intervention and control groups on this outcome variable, neither Froelicher 1994 nor Steinke 2004 reported differences between the groups on this outcome.

Klein 2007 reported proportions of patients who had returned to regular sexual activity (yes/no) at four months follow-up. The study authors reported that: "The proportion of return to regular sexual activity was higher among participants in the treatment group (87 versus $50 \%$ in the control group, $\mathrm{X}^{2}=4.55$, d.f. $=1$, $\mathrm{P}<0.05$ )“. Compared with the control group, participants in the intervention group were statistically significantly more likely to have returned to regular sexual activity at four months followup (relative risk (RR) 1.71, 95\% confidence interval (CI) 1.26 to 2.32; one trial, 92 participants).

In Froelicher 1994, the total number of participants who returned to sexual activity (yes/no) by 12 weeks follow-up of the total 183 participants were 63 (96\% of 66) in the control group and 59 ( $97 \%$ of 61 ) in the intervention group. In a comparison of the intervention and control groups, there were no significant differences on this outcome at 12 weeks follow-up (RR 1.01, 95\% CI 0.94 to 1.09 ; one trial, 127 participants).

In Steinke 2004, there were no significant differences between the experimental and control groups on return to sexual activity at three months follow-up (RR 0.98, 95\% CI 0.88 to 1.10 ; one trial, 
115 participants). There were no differences on this outcome at any time using $\mathrm{Chi}^{2}$ test at one month $\left(\mathrm{Chi}^{2}\right.$ test $\left.=1.33, \mathrm{P}=0.25\right)$ and Fisher's exact test at three and five months $(\mathrm{P}=1.00$ and $\mathrm{P}=$ 0.71 , respectively).

\section{Other outcomes (not prespecified in our protocol)}

One study reported knowledge about sex after an MI as an outcome (Steinke 2004).

Steinke 2004 assessed knowledge about sex after an MI using the 25-item Sex After MI Knowledge Test at baseline and at 1, 3, and 5 months follow-up. No scores were reported, but based on results of a repeated measures ANCOVA of changes in means over the three timepoints, as described above, the study authors concluded: "The experimental group had significantly greater knowledge at one month post $\mathrm{MI}[\mathrm{F}(2,75)=10.47, \mathrm{P}<0.01]$ “. They reported: "There were no significant differences between the two groups [for knowledge] at any other timepoints".

\section{DISCUSSION}

Sexual problems are more commonly reported by men and women with cardiovascular disease than those without cardiovascular disease (Schumann 2010; Kutmeç 2011). Such problems negatively impact on quality of life, psychological well-being, and marital or partnership satisfaction (Traeen 2007; Günzler 2009). Whilst sexual counselling for patients and their partners or spouses has been recommended as an important component of cardiac rehabilitation (Levine 2012; Steinke 2013a), there is currently little evidence on the effectiveness of sexual counselling for improving outcomes. This Cochrane review assessed the effectiveness of sexual counselling for sexual problems among people with cardiovascular disease. We included three studies and 381 participants in total. We considered the interventions within these trials to be too heterogeneous to permit meta-analysis, so we provided a narrative synthesis of the findings in this review.

\section{Summary of main results}

\section{Primary outcomes}

Two of the three included studies reported a sexual function outcome (Steinke 2004; Klein 2007). These studies used sexual function measures that each contained a subscale that measured sexual satisfaction. Klein 2007 reported significantly higher levels of sexual function and satisfaction in the intervention group at one and four months follow-up after sexual counselling in comparison to the control group. There were no differences in these measures between the intervention and control groups in Steinke 2004.

\section{Secondary outcomes}

Klein 2007 was the only included study to assess marital satisfaction as an outcome; the study authors found no significant differences between groups at any of the three timepoints. Steinke 2004 included quality of life as an outcome, and found no significant differences between groups at any of the timepoints. Two studies reported anxiety outcomes (Steinke 2004; Klein 2007); only Steinke 2004 reported significant differences between the groups, with larger increases in anxiety among the intervention group from baseline to one month follow-up than the control group, but no differences at any other timepoint. All three studies reported the number of patients returning to sexual activity following myocardial infarction (MI) as an outcome. While Klein 2007 reported significant differences between intervention and control groups on percentage returning to sexual activity following MI (more participants in the intervention group had returned to sexual activity than in the control group), neither Froelicher 1994 nor Steinke 2004 reported differences between the groups on this outcome. Steinke 2004 reported knowledge about sex after MI as an outcome. Higher levels were reported among the intervention group than the control group at one month follow-up, but not at other timepoints.

None of the included studies reported on partner-reported outcomes.

\section{Overall completeness and applicability of evidence}

Due to the heterogeneous nature of the three included trials and based on the primary and secondary outcomes included in this Cochrane review, there is little evidence on which to base a decision on the overall effectiveness of sexual counselling interventions for people with cardiovascular disease or their partners.

\section{Quality of the evidence}

We judged the overall methodological quality of the included trials to be very low. Details of trial methodology and intervention content were generally poorly reported. For example, Froelicher 1994 and Klein 2007 did not provide any information about the randomisation sequence generation or allocation concealment. Blinding of outcome assessment was not done in Froelicher 1994 or it was unclear whether it was done or not in Klein 2007. All three studies suffered from high risk of attrition bias.

There are always risks of bias associated with outcome measures that rely on self-reporting. All outcomes within this Cochrane review were self-reported measures.

\section{Potential biases in the review process}


We have taken every step to minimise bias in the review process. We performed a systematic and comprehensive search for studies. We strictly adhered to study inclusion criteria, and well-defined data extraction and managements protocols. Two review authors (MB and SD) independently assessed and agreed on all included and excluded studies. There was very little disagreement on inclusion and exclusion of studies. When there was a disagreement, MB and SD discussed it with a third review author (DD) and reached consensus.

\section{Agreements and disagreements with other studies or reviews}

We did not identify any other reviews of sexual counselling for people with cardiovascular disease. The World Health Organization (WHO) recently rated the evidence that 'brief sexuality-related counselling' is more effective than 'usual standard of care' in reducing sexual difficulties and sexual distress as strong (scoring 8.4 out of a possible maximum rating of nine) (WHO 2015). However, most of this evidence comes from research in the context of HIV/AIDS. A previous review of sexual counselling for sexual dysfunction among the general population found that group psychotherapy was more likely than the control group (who received no treatment) to reduce the number of men with "persistence of erectile dysfunction” at post-treatment (Melnik 2007). It would be useful for future research to explore the effectiveness of groupbased sexual counselling interventions tailored to people with cardiovascular disease, as this intervention format is likely to be feasible to deliver as a component of hospital cardiac rehabilitation programmes.

\section{AUTHORS'CONCLUSIONS}

\section{Implications for practice}

Our Cochrane review includes only three studies which assessed the effect of three quite different types of interventions. In addition, all included studies were at high risk of bias. Therefore, our review provides limited evidence to support sexual counselling for sexual problems in people with cardiovascular disease.

One study, Klein 2007, suggested that a high intensity intervention (five hour total duration of sexual therapy of patients and their partners, with clinical social worker and a medical doctor trained in sexual co-therapy) may result in improved sexual outcomes for patients. They reported that patients that received such an intervention were likely to return to sexual activity more quickly following a myocardial infarction (MI), and reported higher levels of sexual function and sexual satisfaction in comparison to control patients that received only usual care. However, there are a number of possible sources of bias within this study, including selection bias, performance bias, and detection bias. Therefore the findings should be treated with some caution. In addition, included participants may not be representative of patients more generally, as they were all recruited through one medical centre. Furthermore, such a resource and time intensive intervention is unlikely to be feasible or practicable in most health service settings.

The two other studies of lower intensity interventions did not report significant benefits to the intervention group in comparison to the control group (Froelicher 1994; Steinke 2004). Steinke 2004 did find some evidence that a 15-minute video improved short term knowledge about resuming sexual activity following a MI among the intervention group. Both of these studies has small sample sizes and were underpowered to detect an effect of the intervention.

\section{Implications for research}

There is a clear need for robust, adequately powered, methodologically rigorous randomised controlled trials (RCTs) of sexual counselling interventions. Such interventions must be pragmatically designed and have the potential to be implemented into general health services. They should be feasible for health service providers to deliver and acceptable to patients and their partners. Group-based sexual counselling interventions tailored to people with cardiovascular disease show some promise (Melnik 2007) and warrant future research. Future studies should describe clearly intervention content and delineate proposed mechanisms of action of interventions, as this will provide insight into which strategies might be most or least effective for people with cardiovascular disease.

\section{ACKNOWLEDGEMENTS}

We acknowledge Ms Milou Fredrix, who screened some of the references for inclusion in this Cochrane review. We also thank Ms Jessie Mulcaire, who extracted some data from included trials. 


\section{R E F E R E N C E S}

\section{References to studies included in this review}

Froelicher 1994 \{published data only\}

Froelicher ES, Kee LL, Newton KM, Lindskog B, Livingston M. Return to work, sexual activity, and other activities after acute myocardial infarction. Heart \& Lung: The Journal of Acute and Critical Care 1994;23(5):423-35.

Klein 2007 \{published data only\}

Klein R, Bar-on E, Klein J, Benbenishty R. The impact of sexual therapy on patients after cardiac events participating in a cardiac rehabilitation program. European Journal of Cardiovascular Prevention \& Rehabilitation 2007;14(5): 672-8.

Steinke 2004 \{published data only\}

Steinke EE, Swan JH. Effectiveness of a videotape for sexual counseling after myocardial infarction. Research in Nursing \& Health 2004;27(4):269-80.

\section{References to studies excluded from this review}

Begot 2013 \{published data only\}

Begot I, Peixoto TCA, Duarte LM, Moreira RSL, Carvalho ACC, Gomes WJ, et al. Impact of an exercise-based rehabilitation program on the sexual function of men after acute myocardial infarction: Randomized controlled trial. Cardiology (Switzerland) 2013;126:92.

Begot 2015 \{published data only\} Begot I, Peixoto TCA, Gonzaga LRA, Bolzan DW, Papa V, Carvalho ACC, et al. A home-based walking program improves erectile dysfunction in men with an acute myocardial infarction. American Journal of Cardiology 2015; 115(5):571-5. [DOI: 10.1016/j.amjcard.2014.12.007]

Belardinelli 2005 \{published data only\}

Belardinelli R, Lacalaprice F, Faccenda E, Purcaro A, Perna G. Effects of short-term moderate exercise training on sexual function in male patients with chronic stable heart failure. International Journal of Cardiology 2005;101(1):83-90.

Berg 2011 \{published data only\}

Berg SK, Svendsen JH, Zwisler AD, Pedersen BD, Preisler P, Siersbaek-Hansen L, et al. COPE-ICD: a randomised clinical trial studying the effects and meaning of a comprehensive rehabilitation programme for ICD recipients -design, intervention and population. BMC Cardiovascular Disorders 2011;11:33.

Cohen 1986 \{published data only\} Cohen JA. Sexual counseling of the patient following myocardial infarction. Critical Care Nurse 1986;6(6):18-9, 22-9.

de Araújo 2009 \{published data only\} de Araújo CG. Sexual activity: an exercise to prevent cardiovascular morbidity and mortality?. Expert Review of Cardiovascular Therapy 2009;7(9):1033-6.

Dougherty 2012 \{published data only\} Dougherty CM, Thompson EA, Kudenchuk PJ. Development and testing of an intervention to improve outcomes for partners following receipt of an implantable cardioverter defibrillator in the patient. Advances in Nursing Science 2012;35(4):359-77.

Fisher 1997 \{published data only\}

Fisher BJ. The effects of an information/counselling session and partner participation in treadmill testing on sexual functioning following myocardial infarction. Dissertation Abstracts International: Section B: The Sciences and Engineering 1997;57:6569.

Johansen 2013 \{published data only\}

Johansen PP, Zwisler AD, Hastrup-Svendsen J, Frederiksen M, Lindschou J, Winkel P, et al. The CopenHeartSF trial - comprehensive sexual rehabilitation programme for male patients with implantable cardioverter defibrillator or ischaemic heart disease and impaired sexual function: protocol of a randomised clinical trial. BMJ Open 2013;3 (11): $\mathrm{e} 003967$.

Kalka 2009 \{published data only\}

Kalka D, Sobieszczań ska M, Pilecki W, Szawrowicz-Pelka T, Marciniak W, Sebzda T, et al. [Evaluation of ambulatory cardiac rehabilitation influence on the intensity of erectile dysfunction in patients with ischemic heart disease]. Polski Merkuriusz Lekarski 2009;27(160):290-5.

\section{Kohn 2000 \{published data only\}}

Kohn CS, Petrucci RJ, Baessler C, Soto DM, Movsowitz C. The effect of psychological intervention on patients' longterm adjustment to the ICD: a prospective study. Pacing and Clinical Electrophysiology 2000;23(4 Pt 1):450-6.

Kushnir 1976 \{published data only\} Kushnir B, Fox K, Tomlinson I, Aber CP. The effect of a pre-discharge consultation on the resumption of work, sexual activity, and driving following acute myocardial infarction. Scandinavian Journal of Rehabilitation Medicine 1976;8:155-9.

\section{Lie 2010 \{published data only\}}

Lie I, Bunch EH, Smeby NAA, Arnesen H, Hamilton G. Coronary artery bypass graft patients experience unmet physical and psychosocial symptoms during their early rehabilitation. Results from the intervention group in a randomized controlled trial. European Heart Journal 2010; 31:229.

Lindau 2011 \{published data only\}

Lindau ST, Abramsohn E, Chan P, Garavalia B, Garavalia L, Krumholz HM, et al. Abstract P147: Sexual problems experienced by women after an MI. Session Title: Poster Session II. 2011 May 13. Circulation: Cardiovascular Quality and Outcomes 2011; Vol. 4:AP147.

Mittag 2006 \{published data only\} Mittag O, China C, Hoberg E, Juers E, Kolenda KD, Richardt G, et al. Outcomes of cardiac rehabilitation with versus without a follow-up intervention rendered by telephone (Luebeck follow-up trial): overall and gender- 
specific effects. International Journal of Rehabilitation Research 2006;29(4):295-302.

Moore 2001 \{published data only\}

Moore SM, Dolansky MA. Randomized trial of a home recovery intervention following coronary artery bypass surgery. Research in Nursing \& Health 2001;24(2):93-104.

Ross 2000 \{published data only\} Ross AB, Brodie EE, Carroll D, Niven CA, Hotchkiss $\mathrm{R}$. The psychosocial and physical impact of exercise rehabilitation following coronary artery bypass surgery. Coronary Health Care 2000;4(2):63-70.

Roviaro 1984 \{published data only\} Roviaro S, Holmes DS, Holmsten RD. Influence of a cardiac rehabilitation program on the cardiovascular, psychological, and social functioning of cardiac patients. Journal of Behavioral Medicine 1984;7(1):61-81.

Steinke 1998 \{published data only\}

Steinke EE, Patterson-Midgley P. Importance and timing of sexual counseling after myocardial infarction. Journal of Cardiopulmonary Rehabilitation 1998;18(6):401-7.

Steinke 2012 \{published data only\}

Steinke E, Mosack V, Hertzog J, Wright DW. A social-cognitive sexual counseling intervention postMI: development and pilot testing. Perspectives in Psychiatric Care 2012;49(3):162-70. [DOI: 10.1111/ j.1744-6163.2012.00345.x]

Sumanen 2005 \{published data only\} Sumanen M, Ojanlatva A, Koskenvuo M, Mattila K. GPs should discuss sex life issues with coronary heart patients. Sexual and Relationship Therapy 2005;20(4):443-52.

Whipple 1987 \{published data only\}

Whipple B. Sexual counseling of couples after a mastectomy or myocardial infarction. Nursing Forum 1987;23(3): $85-91$.

\section{Additional references}

\section{Agren 2009}

Agren S, Frisman GH, Berg S, Svedjeholm R, Strömberg A. Addressing spouses' unique needs after cardiac surgery when recovery is complicated by heart failure. Heart \& Lung: The Journal of Acute and Critical Care 2009;38(4):284-91.

\section{Barnason 2011}

Barnason S, Steinke E, Mosack V, Wright DW. Comparison of cardiac rehabilitation and acute care nurses perceptions of providing sexual counseling for cardiac patients. Journal of Cardiopulmonary Rehabilitation and Prevention 2011;31 (3):157-63

Bertie 1992

Bertie J, King A, Reed N, Marshall A, Ricketts C. Benefits and weakness of a cardiac rehabilitation programme. Journal of the Royal College of Physicians of London 1992;26(2): $147-51$.

\section{Brandão 2014}

Brandão T, Schulz MS, Matos PM. Psychological intervention with couples coping with breast cancer: a systematic review. Psychology \& Health 2014;29(5):

491-516.

\section{Brotto 2008}

Brotto LA, Heiman JR, Goff B, Greer B, Lentz GM, Swisher E, et al. A psychoeducational intervention for sexual dysfunction in women with gynecologic cancer. Archives of Sexual Behavior 2008;37(2):317-29. [DOI: 10.1007/s10508-007-9196-x]

Byrne 2010

Byrne M, Doherty S, McGee HM, Murphy AW. General practitioner views about discussing sexual issues with patients with coronary heart disease: a national survey in Ireland. BMC Family Practice 2010;11:40.

Byrne 2013

Byrne M, Doherty S, Murphy AW, McGee HM, Jaarsma T. The CHARMS Study: cardiac patients' experiences of sexual problems following cardiac rehabilitation. European Journal of Cardiovascular Nursing 2013;12(6):551-9. [DOI 10.1177/1474515113477273]

\section{Clayton 1997}

Clayton AH, McGarvey EL, Clavet GJ. The Changes in Sexual Functioning Questionnaire (CSFQ): development, reliability, and validity. Psychopharmacology Bulletin 1997; 33(4):731-45.

\section{Coyne 2001}

Coyne JC, Rohrbaugh MJ, Shoham V, Sonnega JS, Nicklas JM, Cranford JA. Prognostic importance of marital quality for survival of congestive heart failure. The American Journal of Cardiology 2001;88(5):526-9.

\section{D’Eath 2013}

D'Eath M, Byrne M, Doherty S, McGee H, Murphy AW. The Cardiac Health and Assessment of Relationship Management and Sexuality study: a qualitative inquiry of patient, general practitioner, and cardiac rehabilitation staff views on sexual assessment and counseling for cardiac patients. Journal of Cardiovascular Nursing 2013;28(2) E1-13.

Dalteg 2011

Dalteg T, Benzein E, Fridlund B, Malm D. Cardiac disease and its consequences on the partner relationship: a systematic review. European Journal of Cardiovascular Nursing 2011;10(3):140-9.

\section{Derogatis 1997}

Derogatis LR. The Derogatis Interview for Sexual Functioning (DISF/DISF-SR): an introductory report. Journal of Sex \& Marital Therapy 1997;23(4):291-304. [DOI: 10.1080/00926239708403933]

Djurović 2010

Djurović A, Marić D, Brdareski Z, Konstantinov ć L, Rafajlovski S, Obradović S, et al. Sexual rehabilitation after myocardial infarction and coronary bypass surgery: why do we not perform our job?. Vojnosanitetski Pregled 2010;67(7):579-87. 


\section{Doherty 2011}

Doherty S, Byrne M, Murphy AW, McGee HM. Cardiac rehabilitation staff views about discussing sexual issues with coronary heart disease patients: a national survey in Ireland. European Journal of Cardiovascular Nursing 2011;10(2):1017.

\section{Dong 2011}

Dong J-Y, Zhang Y-H, Qin L-Q. Erectile dysfunction and risk of cardiovascular disease: meta-analysis of prospective cohort studies. Journal of the American College of Cardiology 2011;58(13):1378-85.

Egger 1997

Egger M, Davey Smith G, Schneider M, Minder C. Bias in meta-analysis detected by a simple, graphical test. $B M J$ 1997;315(7109):629-34.

\section{Ferrans 1985}

Ferrans CE, Powers MJ. Quality of life index: development and psychometric properties. Advances in Nursing Science 1985;8(1):15-24.

\section{Fisher 2005}

Fisher WA, Rosen RC, Eardley I, Sand M, Goldstein I. Sexual experience of female partners of men with erectile dysfunction: the Female Experience of Men's Attitudes to Life Events and Sexuality (FEMALES) Study. The Journal of Sexual Medicine 2005;2(5):675-84.

\section{Fowers 1993}

Fowers BJ, Olsen D. ENRICH Marital Satisfaction Scale: a brief research and clinical tool. Journal of Family Psychology 1993;7(2):176-85.

\section{Fridlund 1991}

Fridlund B, Högstedt B, Lidell E, Larsson PA. Recovery after myocardial infarction: Effects of a caring rehabilitation programme. Scandinavian Journal of Caring Sciences 1991;5 (1):23-32.

\section{Froelicher 1994}

Froelicher ES, Kee LL, Newton KM, Lindskog B, Livingston M. Return to work, sexual activity, and other activities after acute myocardial infarction. Heart \& Lung: The Journal of Critical Care 1994;23(5):423-35.

\section{Gamel 1993}

Gamel C, Davis BD, Hengeveld M. Nurses' provision of teaching and counselling on sexuality: a review of the literature. Journal of Advanced Nursing 1993;18(8): 1219-27.

\section{Goossens 2011}

Goossens E, Norekvål TM, Faerch J, Hody L, Olsen SS, Darmer MR, et al. Sexual counselling of cardiac patients in Europe: culture matters. International Journal of Clinical Practice 2011;65(10):1092-9.

\section{Günzler 2009}

Günzler C, Kriston L, Harms A, Berner MM. Association of sexual functioning and quality of partnership in patients in cardiovascular rehabilitation--a gender perspective. The Journal of Sexual Medicine 2009;6(1):164-74.

\section{Harbord 2006}

Harbord RM, Egger M, Sterne JA. A modified test for small-study effects in meta-analyses of controlled trials with binary endpoints. Statistics in Medicine 2006;25(20): 3443-57.

\section{Higgins 2011}

Higgins JPT, Green S (editors). Cochrane Handbook for Systematic Reviews of Interventions Version 5.1.0 [updated March 2011]. The Cochrane Collaboration, 2011. Available from www.cochrane-handbook.org.

\section{Hoekstra 2012a}

Hoekstra T, Lesman-Leegte I, Couperus MF, Sanderman R, Jaarsma $T$. What keeps nurses from the sexual counselling of patients with heart failure?. Heart \& Lung: The Journal of Acute and Critical Care 2012;41(5):492-9.

\section{Hoekstra 2012b}

Hoekstra T, Lesman-Leegte I, Luttik M, Sanderman R, van Veldhuisen D, Jaarsma T. Sexual problems in elderly male and female patients with heart failure. Heart 2012;98(22): $1647-52$.

\section{Hoga 2010}

Hoga LA, Tiburcio CA, Borges AL, Zoboli EL, ChavezAlvarez RE. Counseling regarding sexual and reproductive behavior: principles and practices of Catholic priests. Revista Latino-Americana de Enfermagen 2010;18(6): 1237-45.

\section{Ivarsson 2009}

Ivarsson B, Fridlund B, Sjöberg T. Information from health care professionals about sexual function and coexistence after myocardial infarction: a Swedish national survey. Heart \& Lung: The Journal of Critical Care 2009;38(4): $330-5$.

\section{Ivarsson 2010}

Ivarsson B, Fridlund B, Sjöberg T. Health professionals' views on sexual information following MI. British Journal of Nursing 2010;19(16):1052-4.

\section{Jaarsma 2010a}

Jaarsma T. Addressing sexual function in cardiac patients: do we need guidelines? Foreword. Journal of Cardiovascular Nursing 2010;25(2):149-50.

\section{Jaarsma 2010b}

Jaarsma T, Steinke EE, Gianotten WL. Sexual problems in cardiac patients: how to assess, when to refer. Journal of Cardiovascular Nursing 2010;25(2):159-64.

\section{Jaarsma 2010c} Jaarsma T, Strömberg A, Frindlund B, De Geest $S$, Mårtensson J, Moons P, et al. Sexual counselling of cardiac patients: nurses' perception of practice, responsibility and confidence. European Journal of Cardiovascular Nursing 2010;9(1):24-9.

Katz 2007

Katz A. Sexuality and myocardial infarction. American Journal of Nursing 2007;107(3):49-52.

\section{Kazemi-Saleh 2008}

Kazemi-Saleh D, Pishgoo B, Farrokhi F, Fotros A, Assari S. Sexual function and psychological status among males 
and females with ischemic heart disease. Journal of Sexual Medicine 2008;5(10):2330-7.

\section{Keller 2006}

Keller A, McGarvey EL, Clayton AH. Reliability and construct validity of the Changes in Sexual Functioning Questionnaire short-form (CSFQ-14). Journal of Sex \& Marital Therapy 2006;32(1):43-52. [DOI: 10.1080/ 00926230500232909]

\section{Klein 2007}

Klein R, Bar-on E, Klein J, Benbenishty R. The impact of sexual therapy on patients after cardiac events participating in a cardiac rehabilitation program. European Journal of Cardiovascular Prevention and Rehabilitation 2007;14(5): 672-8.

Kriston 2010

Kriston L, Günzler C, Agyemang A, Bengel J, Berner MM, SPARK Study Group. Effect of sexual function on healthrelated quality of life mediated by depressive symptoms in cardiac rehabilitation. Findings of the SPARK project in 493 patients. Journal of Sexual Medicine 2010;7(6): 2044-55.

\section{Kushnir 1976}

Kushnir B, Fox KM, Tomlinson W, Aber CP. The effect of a pre-discharge consultation on the resumption of work, sexual activity, and driving following acute myocardial infarction. Scandinavian Journal of Rehabilitation Medicine 1976;8:155-9.

\section{Kutmeç 2011}

Kütmeç C, Yurtsever S. Effects of sexual function of essential hypertensions in women. European Journal of Cardiovascular Nursing 2011;10(1):56-63.

\section{Lassen 2013}

Lassen B, Gattinger H, Saxer S. A systematic review of physical impairments following radical prostatectomy: effect of psychoeducational interventions. Journal of Advanced Nursing 2013;69(12):2602-12.

\section{Lefebvre 2011}

Lefebvre C, Manheimer E, Glanville J. Chapter 6: Searching for studies. In: Higgins JPT, Green $S$ (editors) Cochrane Handbook for Systematic Reviews of Interventions Version 5.1.0 [updated March 2011]. The Cochrane Collaboration, 2011. Available from www.cochrane-handbook.org. The Cochrane Collaboration.

\section{Levine 2012}

Levine GN, Steinke EE, Bakaeen FG, Bozkurt B, Cheitlin $\mathrm{MD}$, Conti JB, et al. Sexual activity and cardiovascular disease: A scientific statement from the American Heart Association. Circulation 2012;125(8):1058-72.

\section{Lue 2004}

Lue TF, Giuliano F, Montorsi F, Rosen RC, Andersson KE, Althof $S$, et al. Summary of the recommendations on sexual dysfunctions in men. The Journal of Sexual Medicine 2004; 1(1):6-23.

\section{McGahuey 2000}

McGahuey CA, Gelenberg AJ, Laukes CA, Moreno FA, Delgado PL, McKnight KM, et al. The Arizona Sexual
Experience Scale (ASEX): reliability and validity. Journal of Sex \& Marital Therapy 2000;26(1):25-40. [DOI: 10.1080/ 009262300278623]

\section{Melnik 2007}

Melnik T, Soares BG, Nasello AG. Psychosocial interventions for erectile dysfunction. Cochrane Database of Systematic Reviews 2007, Issue 3. [DOI: 10.1002/ 14651858.CD004825.pub2]

\section{Meston 2005}

Meston C, Trapnell P. Development and validation of a fivefactor sexual satisfaction and distress scale for women: the Sexual Satisfaction Scale for Women (SSS-W). The Journal of Sexual Medicine 2005;2(1):66-81.

\section{Miles 2007}

Miles C, Candy B, Jones L, Williams R, Tookman A, King M. Interventions for sexual dysfunction following treatments for cancer. Cochrane Database of Systematic Reviews 2007, Issue 4. [DOI: 10.1002/14651858.CD005540.pub2]

\section{Mosack 2009}

Mosack V, Steinke EE. Trends in sexual concerns after myocardial infarction. Journal of Cardiovascular Nursing 2009;24(2):162-70.

\section{Mulat 2010}

Mulat B, Arbel Y, Mashav N, Saar N, Steinvil A, Heruti R, et al. Depressive symptoms and erectile dysfunction in men with coronary artery disease. Urology 2010;75(1):104-7.

\section{Mykletun 2006}

Mykletun A, Dahl AA, O'Leary MP, Fosså SD. Assessment of male sexual function by the Brief Sexual Function Inventory. BJU International 2006;97(2):316-23.

\section{O' Farrell 2000}

O'Farrell P, Murray J, Hotz SB. Psychologic distress among spouses of patients undergoing cardiac rehabilitation. Heart \& Lung: The Journal of Critical Care 2000;29(2):97-104.

\section{O'Connor 2008}

O'Connor DB, Corona G, Forti G, Tajar A, Lee DM, Finn JD, et al. Assessment of sexual health in aging men in Europe: development and validation of the European Male Ageing Study Sexual Function Questionnaire. The Journal of Sexual Medicine 2008;5(6):1374-85.

\section{O’Donovan 2007}

O'Donovan K. Addressing the taboos: resuming sexual activity after myocardial infarction. British Journal of Cardiac Nursing 2007;2(4):165-74.

\section{Pouraboli 2010}

Pouraboli B, Azizzadeh FM, Mohammad AS. Knowledge and attitudes of nurses in sexual activity and educate it to patients with myocardial infarction and their spouses. Iranian Journal of Critical Care Nursing 2010;2(4):5-6.

\section{Quirk 2002}

Quirk FH, Heiman JR, Rosen RC, Laan E, Smith MD, Boolell M. Development of a sexual function questionnaire for clinical trials of female sexual dysfunction. Journal of Women's Health \& Gender-Based Medicine. 2002;11(3): 277-89. 
RevMan 2014 [Computer program]

The Nordic Cochrane Centre, The Cochrane Collaboration. Review Manager (RevMan). Version 5.3. Copenhagen: The Nordic Cochrane Centre, The Cochrane Collaboration, 2014.

Rosen 1997

Rosen RC, Riley A, Wagner G, Osterloh IH, Kirkpatrick J, Mishra A. The International Index of Erectile Function (IIEF): a multidimensional scale for assessment of erectile dysfunction. Urology 1997;49(6):822-30.

Rosen 2000

Rosen C, Brown J, Heiman S, Leiblum C, Meston R, Shabsigh D, et al. The Female Sexual Function Index (FSFI): a multidimensional self-report instrument for the assessment of female sexual function. Journal of Sex \& Marital Therapy 2000;26(2):191-208. [DOI: 10.1080/ 009262300278597]

\section{Schumann 2010}

Schumann J, Zellweger MJ, Di Valentino M, Piazzalonga S, Hoffmann A. Sexual dysfunction before and after cardiac rehabilitation. Rehabilitation Research and Practice 2010; 2010:823060. [DOI: 10.1155/2010/823060]

\section{Schwarz 2008}

Schwarz E, Kapur V, Bionat S, Rastogi S, Gupta R, Rosanio $S$. The prevalence and clinical relevance of sexual dysfunction in women and men with chronic heart failure. International Journal of Impotence Research 2008;20:85-91.

\section{Song 2011}

Song H, Oh H, Kim H, Seo W. Effects of a sexual rehabilitation intervention program on stroke patients and their spouses. NeuroRehabilitation 2011;28(2):143-50.

\section{Speilberger 1983}

Speilberger CD. State-Trait Anxiety Inventory. Consulting Psychologists 1983.

\section{Steinke 1998}

Steinke E, Patterson-Midgley P. Importance and timing of sexual counselling after myocardial infarction. Journal of Cardiopulmonary Rehabilitation 1998;18(6):401-7.

\section{Steinke 2004}

Steinke EE, Swan JH. Effectiveness of a videotape for sexual counseling after myocardial infarction. Research in Nursing \& Health 2004;27(4):269-80.

\section{Steinke 2011a}

Steinke EE, Barnason S, Mosack V, Wright DW. Changes in myocardial infarction-specific sexual counseling by cardiac nurses. Dimensions of Critical Care Nursing 2011;30(6): $331-8$.

\section{Steinke 2011b}

Steinke EE, Mosack V, Barnason S, Wright DW. Progress in sexual counselling by cardiac nurses, 1994 to 2009. Heart \& Lung: The Journal of Acute and Critical Care 2011;40(3): e15-24.

Steinke 2013a

Steinke EE, Jaarsma T, Barnason SA, Byrne M, Doherty S, Dougherty CM, et al. Sexual counseling for individuals with cardiovascular disease and their partners: a consensus document from the American Heart Association and the ESC Council on Cardiovascular Nursing and Allied Professions (CCNAP). Circulation 2013;128(18):2075-96. [DOI: 10.1161/CIR.0b013e31829c2e53]

\section{Steinke 2013b}

Steinke EE, Mosack V, Hill TJ. Sexual self-perception and adjustment of cardiac patients: a psychometric analysis. Journal of Research in Nursing 2013;18(3):191-201.

\section{Syrjala 2000}

Syrjala KL, Schroeder TC, Abrams JR, Atkins TZ, Brown WS, Sanders JE, et al. Sexual function measurement and outcomes in cancer survivors and matched controls. The Journal of Sex Research 2000;37(3):213-25.

\section{Taylor 1994}

Taylor JF, Rosen RC, Leiblum SR. Self-report assessment of female sexual function: psychometric evaluation of the Brief Index of Sexual Functioning for Women. Archives of Sexual Behavior 1994;23(6):627-43.

Taylor 2011

Taylor A, Gosney MA. Sexuality in older age: essential considerations for healthcare professionals. Age and Ageing 2011;40(5):538-43.

Traeen 2007

Traeen B, Olsen S. Sexual dysfunction and sexual well-being in people with heart disease. Sexual \& Relationship Therapy 2007;22(2):193-208.

\section{Ware 1992}

Ware JE Jr, Sherbourne CD. The MOS 36-item short-form health survey (SF-36). 1. Conceptual framework and item selection. Medical Care 1992;30(6):473-83.

\section{Ware 1995}

Ware JE Jr, Kosinski M, Keller SD. A 12-item short-form health survey: construction of scales and preliminary tests of reliability and validity. Medical Care 1995;34(3):220-33.

\section{WHO 2011}

World Health Organization. Global atlas on cardiovascular disease prevention and control. http://apps.who.int/iris/ handle/10665/44701 (accessed 1 January 2016). Geneva: World Health Organization, 2011.

\section{WHO 2015}

World Health Organization. Brief sexuality-related communication: recommendations for a public health approach. http://www.ncbi.nlm.nih.gov/books/ NBK311023/ (accessed 1 January 2016) 2015.

$Y_{1}$ Id z 2012

$Y_{1} l_{1}$ z H, Dereli E. The views and attitudes of nurses on the topic of sexual counselling. Sexuality and Disability 2012;30(1):89-95.

\section{References to other published versions of this review}

\section{Byrne 2014}

Byrne M, Doherty S, Fridlund BGA, Mårtensson J, Steinke EE, Jaarsma T, Devane D. Sexual counselling for sexual 
problems in patients with cardiovascular disease. Cochrane Database of Systematic Reviews 2014, Issue 2. [DOI:

10.1002/14651858.CD010988]

* Indicates the major publication for the study 


\section{CHARACTERISTICS OF STUDIES}

\section{Characteristics of included studies [ordered by study ID]}

\section{Froelicher 1994}

\section{Methods}

Study design: randomised controlled trial (RCT) with 3 groups. We only compared 2 groups in this review

Data collection timepoints: 3 data collection timepoints: Time 1, baseline (prior to intervention); Time 2, 12 week follow-up; Time 3, 24 week follow-up
Setting: participants were recruited on admission to coronary care units of 7 Seattle hospitals, USA

\section{Inclusion criteria}

- Primary diagnosis of confirmed acute myocardial infarction, free from complications for a minimum of 24 hours.

- Able to walk without aid.

- Under 71 years of age.

- Able to speak and read English.

- Free from serious non-cardiac complications before this admission.

- Resided within a 50 mile radius from the University of Washington.

Exclusion criteria: none documented.

Participants randomised: 258 participants in total; 219 men and 39 women, randomised to 3 groups

Group 1: 84 patients were randomly assigned to the control group (usual care); Group 2: 88 patients were randomly assigned to the first intervention group (usual care plus exercise); Group 3: 86 patients were randomly assigned to the intervention group (usual care plus exercise plus education-counselling intervention)

As we were interested in groups 2 and 3 for our comparison (see below), we included a total of 174 patients for this review
Interventions
Control: this study had two comparison groups.

- Received usual care only, which involved conventional medical and nursing management throughout all phases of hospitalisation and convalescence at home.

- Received usual care plus an exercise programme which was delivered during the 3 months following discharge from hospital. Patients in this group received exercise prescriptions based on treadmill test results before discharge from hospital. For 3 months following discharge from the hospital, the patients attended weekly 30 minute outpatient appointments with a research nurse and a physical or occupational therapist. During these sessions, the prior week's activities were reviewed, and patients were given a renewed exercise prescription.

As we were interested in the added benefit of the sexual education-counselling intervention, in our review we treated the group that received usual care plus an exercise programme as the control group

Intervention: patients in the intervention group received usual care, plus an exercise intervention described above (as outlined for the control group above), plus they attended a series of 12 1-hour group educational counselling sessions. One of these sessions included education-counselling on resumption of sexual activity following a MI. Other topics covered within the series of education-counselling sessions were: cardiac anatomy, physiology of MI, coronary artery disease risk factors, nutrition, dietary changes, activi- 
ties and exercise, stress and relaxation, issues relating to resumption of work, and emotional reactions to MI. The intervention was delivered to groups of patients. Spouses and friends were encouraged to attend the sessions to assist with problem solving, develop knowledge and skills, and provide family support. Research staff cardiovascular clinical nurse specialists delivered the intervention

\begin{tabular}{|c|c|}
\hline Outcomes & $\begin{array}{l}\text { Outcomes relevant to this review } \\
\text { - One item of the Activity Summary Questionnaire: 'Have you resumed sexual } \\
\text { activity? Yes or no'. }\end{array}$ \\
\hline
\end{tabular}

Notes

The study was conducted between 1977 and 1979, yet this analysis was conducted and reported in 1994

\section{Risk of bias}

\begin{tabular}{|c|c|c|}
\hline Bias & Authors' judgement & Support for judgement \\
\hline $\begin{array}{l}\text { Random sequence generation (selection } \\
\text { bias) }\end{array}$ & Unclear risk & $\begin{array}{l}\text { No details given as to how people were al- } \\
\text { located into each of the three groups }\end{array}$ \\
\hline Allocation concealment (selection bias) & Unclear risk & No detail provided on process. \\
\hline $\begin{array}{l}\text { Blinding of participants and personnel } \\
\text { (performance bias) } \\
\text { All outcomes }\end{array}$ & High risk & $\begin{array}{l}\text { There was no blinding of participants and } \\
\text { study personnel, which may have had an } \\
\text { impact on outcomes }\end{array}$ \\
\hline $\begin{array}{l}\text { Blinding of outcome assessment (detection } \\
\text { bias) } \\
\text { All outcomes }\end{array}$ & High risk & $\begin{array}{l}\text { Research nurses were involved in interven- } \\
\text { tion delivery as well as data collection }\end{array}$ \\
\hline $\begin{array}{l}\text { Incomplete outcome data (attrition bias) } \\
\text { All outcomes }\end{array}$ & High risk & $\begin{array}{l}\text { There was a } 25 \% \text { attrition rate at } 24 \text { weeks } \\
\text { follow-up. }\end{array}$ \\
\hline Selective reporting (reporting bias) & Low risk & $\begin{array}{l}\text { The protocol was unavailable. However, } \\
\text { the study authors reported all the primary } \\
\text { outcome variables in the results section }\end{array}$ \\
\hline Other bias & High risk & $\begin{array}{l}\text { High risk of performance bias. In this trial, } \\
\text { it is likely that exposure to a wide range of } \\
\text { other additional elements }\end{array}$ \\
\hline
\end{tabular}


- Patient underwent recent myocardial infarction or coronary artery bypass grafting and admitted to Phase 2 cardiac rehabilitation programme.

- Under 71 years of age.

Exclusion criteria: women.

Participants randomised: 92 participants in total; all men. 47 patients were assigned to the intervention group and 45 were assigned to the control group

Control: usual care, which was a multidisciplinary phase 2 cardiac rehabilitation programme

Intervention: usual care plus sexual treatment and counselling. The sexual counselling involved three meetings (total 5 hours) between the therapist(s) and the patient. The intervention was delivered by a clinical social worker and medical doctor who specialised in sexual cotherapy. Participants were given the option of inviting their partners to participate. Partners were involved through the 'homework' assignments and exercises The counselling sessions involved the following content:

Session 1: Therapists interviewed patients and introduced themselves and the programme. Patients received a presentation on education around issues related to sexual activity in general and after Myocardial Infarction and coronary artery bypass grafting in particular, including a review of current, effective interventions. Instructions, assignments, and sensate focusing exercises were administered

Session 2: Discussion of experience with the exercises, cognitive behavioural techniques, additional medical checks where necessary, and where necessary, prescription of Sildenafil. $37 \%$ of those in intervention and $6.7 \%$ of those in the control were prescribed this drug during the study

Session 3: Cognitive behavioural interventions, assessment of progress, and instruction around future sexual activities

\section{Outcomes relevant to this review}

- Return to regular sexual activity.

- Sexual function for cardiac patient questionnaire, to assess sexual function and problems. Compilation of items from several sources, including the International Index of Erectile Dysfunction. Includes items: fear to have sex; desire; confidence in maintaining an erection; satisfactory sexual relationship; sexual pleasure; frequency of erection; erection solid enough for penetration; satisfaction in frequency of sex; premature ejaculation; achieving orgasm; frequency of satisfaction in sexual intercourse; health problems during sexual relations.

- Anxiety: Speilberger's Anxiety Scale to measure state anxiety.

- Marital satisfaction: Olson's Enrich Marital Satisfaction. proportion with coronary artery bypass grafting and previous myocardial infarction. More intervention patients were post- coronary artery bypass grafting ( $44 \%$ versus $27 \%$, $\mathrm{P}<0.001)$ and more patients in the control group were post-myocardial infarction $(82 \%$ 
Klein 2007 (Continued)

versus $60 \%, \mathrm{P}<0.05$ )

\section{Risk of bias}

\begin{tabular}{l|l|l} 
Bias & Authors' judgement & Support for judgement \\
\hline $\begin{array}{l}\text { Random sequence generation (selection } \\
\text { bias) }\end{array}$ & Unclear risk & $\begin{array}{l}\text { The trial gave no information on how par- } \\
\text { ticipants were assigned to groups }\end{array}$ \\
\hline Allocation concealment (selection bias) & Unclear risk & No detail given on the process. \\
\hline $\begin{array}{l}\text { Blinding of participants and personnel } \\
\text { (performance bias) } \\
\text { All outcomes }\end{array}$ & High risk & $\begin{array}{l}\text { There was no blinding of participants and } \\
\text { study personnel, which may have had an } \\
\text { impact on outcomes }\end{array}$ \\
\hline
\end{tabular}

Blinding of outcome assessment (detection Unclear risk bias)

The trial gave limited details about how data were collected: "measurements were based on self-report questionnaires, in addition to information from the patient's medical file“

Incomplete outcome data (attrition bias) High risk All outcomes

Unclear number of participants that completed the range of indices of questionnaire. For all reported variables, there was at least $20 \%$ missing data

Selective reporting (reporting bias) Low risk

The protocol was unavailable. However, the study authors reported all the primary outcome variables in the results section

Other bias

High risk

The study authors reported significant differences between the treatment and control groups regarding the proportions of patients with coronary artery bypass grafting and previous myocardial infarction. They suggested that future studies should stratify for these pre-existing characteristics

Steinke 2004

Methods

Study design: randomised controlled trial (RCT) with 2 groups.

Data collection timepoints: 4 data collection timepoints: Time 1 , Pretest, during patients' hospital stay; Time 2, 1 month post myocardial infarction; Time 3, 3 months post myocardial infarction; T4 5 months post myocardial infarction

Participants

Setting: cardiac rehabilitation programme which is part of a large medical centre in the Midwest USA

Inclusion criteria: patients were identified and referred for inclusion by manager of 
cardiac rehabilitation. Inclusion criteria included a diagnosis of myocardial infarction; ability to read, write and speak English; over 30 years of age; sexually active

Exclusion criteria: previous psychiatric problems; discharged to a nursing home; complex, multiple problems

Participants randomised: 115 participants in total; 92 men and 23 women. 64 assigned to intervention condition and 51 assigned to the control condition

Interventions

Control: usual care, which involved receiving standard verbal and written instructions provided by the cardiac rehabilitation staff about resuming sexual activity following a myocardial infarction. This contained brief guidelines about resuming sexual activity. "Verbal discussion on resuming sexual activities varied depending on time constraints and comfort with the topic by the cardiac rehabilitation nurse"

Intervention: usual care, plus a 15-minute video. The video was developed by Steinke and reviewed by clinical experts. The intervention contained content on: the effect of the heart attack on sexuality and sexual function, communicating with the partner, the impact of cardiac risk factors on sexual function, specific suggestions on when and how to resume sexual activity, and the effects of various medications on sexual function

Outcomes

Notes

\section{Outcomes relevant to this review}

- Anxiety: Speilbergers State Trait Anxiety Inventory.

- Quality of Life: Ferrans \& Powers Quality of Life Index (QLI) - Cardiac Version III.

- Knowledge: 25-item Sex After myocardial infarction Knowledge Test.

- Sexual function: The Watts Sexual Function Questionnaire (WSFQ) with 4 subscales: sexual desire; arousal; orgasm; and satisfaction.

- Return to sexual activity: 2 questions: was the patient sexually active and when they had returned to sexual activity.

Risk of bias

\begin{tabular}{l|l|l}
\hline Bias & Authors' judgement & Support for judgement \\
\hline $\begin{array}{l}\text { Random sequence generation (selection } \\
\text { bias) }\end{array}$ & Low risk & $\begin{array}{l}\text { Random assignment to groups was accom- } \\
\text { plished by a coin toss. }\end{array}$ \\
\hline $\begin{array}{l}\text { Allocation concealment (selection bias) } \\
\text { Blinding of participants and personnel } \\
\text { (performance bias) } \\
\text { All outcomes }\end{array}$ & High risk & The trial gave no details on the process. \\
\hline
\end{tabular}

Blinding of outcome assessment (detection Unclear risk bias)

All outcomes
$45 \%$ attrition rate in intervention group, and $27 \%$ in the control group
High risk
The trial gave no details. 
Steinke 2004 (Continued)

\begin{tabular}{l|l|l}
\hline Selective reporting (reporting bias) & Low risk & $\begin{array}{l}\text { The trial authors reported all variables in } \\
\text { the results section }\end{array}$ \\
\hline Other bias & High risk & $\begin{array}{l}\text { Threat to external validity. The manager } \\
\text { of cardiac rehabilitation identified and re- } \\
\text { ferred participants. Therefore participants } \\
\text { are unlikely to be representative of the gen- } \\
\text { eral population. It was largely a white, mar- } \\
\text { ried, educated sample }\end{array}$ \\
\hline
\end{tabular}

Abbreviations: CABG: coronary artery bypass grafting; RCT: randomised controlled trials; MI: myocardial infarction.

Characteristics of excluded studies [ordered by study ID]

\begin{tabular}{|c|c|}
\hline Study & Reason for exclusion \\
\hline Begot 2013 & The intervention did not match the inclusion criteria of this Cochrane review \\
\hline Begot 2015 & The intervention did not match the inclusion criteria of this Cochrane review \\
\hline Belardinelli 2005 & The intervention did not match the inclusion criteria of this Cochrane review \\
\hline Berg 2011 & Not a randomised controlled trial (RCT) of an intervention (protocol only, study ongoing) \\
\hline Cohen 1986 & Not a RCT of an intervention. \\
\hline de Araújo 2009 & Not a RCT of an intervention. \\
\hline Dougherty 2012 & Not a RCT of an intervention. \\
\hline Fisher 1997 & Not a RCT of an intervention. \\
\hline Johansen 2013 & Not a RCT of an intervention (protocol only, study ongoing). \\
\hline Kalka 2009 & The intervention did not match the inclusion criteria of this Cochrane review \\
\hline Kohn 2000 & The intervention did not match the inclusion criteria of this Cochrane review \\
\hline Kushnir 1976 & Not a RCT of an intervention. \\
\hline Lie 2010 & $\begin{array}{l}\text { The intervention does not appear to match the inclusion criteria. We were only able to obtain the abstract and } \\
\text { were unable to obtain full information despite contacting the study author }\end{array}$ \\
\hline Lindau 2011 & Not a RCT of an intervention. \\
\hline
\end{tabular}


(Continued)

Mittag 2006 The intervention did not match the inclusion criteria of this Cochrane review

Moore 2001 The intervention did not match the inclusion criteria of this Cochrane review

Ross $2000 \quad$ The intervention did not match the inclusion criteria of this Cochrane review

Roviaro 1984 The intervention did not match the inclusion criteria of this Cochrane review

Steinke $1998 \quad$ Not a RCT of an intervention.

Steinke $2012 \quad$ Not a RCT of an intervention.

Sumanen 2005 Not a RCT of an intervention.

Whipple 1987 Not a RCT of an intervention.

Abbreviations: RCT: randomised controlled trial. 


\section{DATA ANDANALYSES}

This review has no analyses.

\section{A P P E N D I C E S}

\section{Appendix I. Electronic database search strategies}

\section{CENTRAL}

\#1 MeSH descriptor: [Sex Counseling] this term only \#2 MeSH descriptor: [Sexual Behavior] this term only \#3 MeSH descriptor: [Sexual Dysfunctions, Psychological] explode all trees \#4 MeSH descriptor: [Sexual Dysfunction, Physiological] explode all trees \#5 MeSH descriptor: [Libido] this term only \#6 sex* near/4 (counsel* or psycholog* or therap*) \#7 sex* near/4 (help* or advi* or inform* or guid* or skill*) \#8 sex* near/4 (problem* or concern* or difficult*) \#9 sex* near/4 (interest* or wish* or activ* or behav* or libido) \#10 (sex* or erect* or libido) near/2 (function* or dysfunction* or disorder*) $\# 11$ (sex* or intima*) near/4 (relation* or spouse* or partner*) $\# 12 \# 1$ or $\# 2$ or $\# 3$ or $\# 4$ or $\# 5$ or \#6 or $\# 7$ or $\# 8$ or $\# 9$ or $\# 10$ or \#11 $\# 13 \mathrm{MeSH}$ descriptor: [Cardiovascular Diseases] explode all trees \#14 cardio* $^{*}$

$\# 15$ cardia* \#16 heart* \#17 coronary* \#18 angina* \#19 myocard $^{*}$ $\# 20$ isch?em* \#21 arrhythmi* \#22 tachycardi* \#23 fibrillat* $\# 24 \# 13$ or \#14 or \#15 or \#16 or \#17 or \#18 or \#19 or \#20 or \#21 or \#22 or \#23 \#25 \#12 and \#24

\section{MEDLINE OVID}

1. Sex Counseling/

2. Sexual Behavior/

3. exp Sexual Dysfunctions, Psychological/

4. exp Sexual Dysfunction, Physiological/

5. Libido/

6. (sex* adj4 (counsel* or psycholog* or therap*)).tw.

7. (sex ${ }^{*}$ adj4 (help* or advi* or inform* or guid* or skill $\left.{ }^{*}\right)$ ).tw.

8. (sex* adj4 (problem* or concern* or difficult $\left.{ }^{*}\right)$ ).tw.

9. (sex* adj4 (interest* or wish* or activ* or behav* or libido)).tw.

10. ((sex* or erect* or libido) adj2 (function* or dysfunction* or disorder*)).tw.

11. ((sex* or intima*) adj4 (relation* or spouse* or partner*)).tw.

Sexual counselling for sexual problems in patients with cardiovascular disease (Review)

Copyright (c) 2016 The Cochrane Collaboration. Published by John Wiley \& Sons, Ltd. 
12. or/1-11

13. exp Cardiovascular Diseases/

14. cardio*.tw.

15. cardia*.tw.

16. heart*.tw.

17. coronary*.tw.

18. angina* ${ }^{*}$ tw.

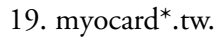

20. isch?em*.tw.

21. arrhythmi*.tw.

22. tachycardi*.tw.

23. fibrillat*.tw.

24. or/13-23

25. 12 and 24

26. randomized controlled trial.pt.

27. controlled clinical trial.pt.

28. randomized.ab.

29. placebo.ab.

30. clinical trials as topic.sh.

31. randomly.ab.

32. trial.ti.

33.26 or 27 or 28 or 29 or 30 or 31 or 32

34. exp animals/ not humans.sh.

35.33 not 34

36. 25 and 35

\section{EMBASE OVID}

1. sexual counseling/

2. sexual behavior/

3. exp psychosexual disorder/

4. exp sexual dysfunction/

5. (sex* adj4 (counsel* or psycholog* or therap*)).tw.

6. (sex* adj4 (help* or advi* or inform* or guid* or skill*)).tw.

7. (sex* adj4 (problem* or concern* or difficult*)).tw.

8. (sex* adj4 (interest* or wish* or activ* or behav* or libido)).tw.

9. ((sex* or erect* or libido) adj2 (function* or dysfunction* or disorder*)).tw.

10. ((sex* or intima*) adj4 (relation* or spouse* or partner*)).tw.

11. or/1-10

12. exp cardiovascular disease/

13. cardio*.tw.

14. cardia*.tw.

15. heart*.tw.

16. coronary*.tw.

17. angina*.tw.

18. myocard*.tw.

19. isch?em*.tw.

20. arrhythmi*.tw.

21. tachycardi*.tw.

22. fibrillat*.tw.

23. or/ $12-22$

24. 11 and 23

25. random \$.tw.

Sexual counselling for sexual problems in patients with cardiovascular disease (Review)

Copyright $\odot 2016$ The Cochrane Collaboration. Published by John Wiley \& Sons, Ltd. 
26. factorial\$.tw.

27. crossover $\$ . t w$.

28. cross over $\$$.tw.

29. cross-over\$.tw.

30. placebo $\$$.tw.

31. (doubl\$ adj blind\$).tw.

32. (singl\$ adj blind $\$$ ).tw.

33. assign $\$$.tw.

34. allocat\$.tw.

35. volunteer\$.tw.

36. crossover procedure/

37. double blind procedure/

38. randomized controlled trial/

39 . single blind procedure/

40.25 or 26 or 27 or 28 or 29 or 30 or 31 or 32 or 33 or 34 or 35 or 36 or 37 or 38 or 39

41. (animal/ or nonhuman/) not human/

42. 40 not 41

43. 24 and 42

44. limit 43 to embase

\section{CINAHL}

S27 S14 AND S26

S26 S15 OR S16 OR S17 OR S18 OR S19 OR S20 OR S21 OR S22 OR S23 OR S24 OR S25

S25 TX allocat* random*

S24 (MH "Quantitative Studies")

S23 (MH "Placebos")

S22 TX placebo*

S21 TX random* allocat*

S20 (MH "Random Assignment")

S19 TX randomi* control* $^{*}$ trial $^{*}$

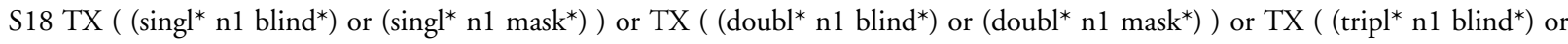
$\left(\right.$ tripl$^{*} \mathrm{n} 1$ mask $\left.\left.^{*}\right)\right)$ or TX $\left(\left(\right.\right.$ trebl$^{*} \mathrm{n} 1$ blind $\left.^{*}\right)$ or $\left(\operatorname{trebl}^{*} \mathrm{n} 1\right.$ mask $\left.\left.^{*}\right)\right)$

S17 TX clinic* $n 1$ trial*

S16 PT Clinical trial

S15 (MH "Clinical Trials+")

S14 S10 AND S13

S13 S11 OR S12

S12 (cardio* or cardia* or heart* or coronary* or angina* or myocard* or ischem* or ischaem* or arrhythmi* or tachycardi* or fibrillat*)

S11 (MH "Cardiovascular Diseases+")

S10 S1 OR S2 OR S3 OR S4 OR S5 OR S6 OR S7 OR S8 OR S9

S9 ((sex* or intima*) N4 (relation* or spouse* or partner*))

S8 ((sex* or erect* or libido) N2 (function* or dysfunction* or disorder*))

S7 (sex* N4 (interest* or wish* or activ* or behav* or libido))

S6 ( $\operatorname{sex}^{*} \mathrm{~N} 4$ (problem* or concern* or difficult*))

S5 ( $\operatorname{sex}^{*}$ N4 (help* or advi* or inform* or guid* or skill*))

S4 ( $\operatorname{sex}^{*} \mathrm{~N} 4$ (counsel* or psycholog* or therap*))

S3 (MH "Sexual Dysfunction, Male+") OR (MH "Sexual Dysfunction, Female+")

S2 (MH "Psychosexual Disorders+")

S1 (MH "Sexual Counseling")

\section{PsycINFO OVID}

Sexual counselling for sexual problems in patients with cardiovascular disease (Review)

Copyright $\odot 2016$ The Cochrane Collaboration. Published by John Wiley \& Sons, Ltd. 
1. sex therapy/

2. exp psychosexual behavior/

3. exp sexual function disturbances/

4. libido/

5. sex drive/

6. (sex* adj4 (counsel* or psycholog* or therap*)).tw.

7. (sex* adj4 (help* or advi* or inform* or guid* or skill*)).tw.

8. (sex* adj4 (problem* or concern* or difficult $\left.{ }^{*}\right)$ ).tw.

9. (sex* adj4 (interest* or wish* or activ* or behav* or libido)).tw.

10. ((sex* or erect* or libido) adj2 (function* or dysfunction* or disorder*)).tw.

11. ((sex* or intima*) adj4 (relation* or spouse* or partner*)).tw.

12. or/ $1-11$

13. exp Cardiovascular Disorders/

14. cardio*.tw.

15. cardia*.tw.

16. heart*.tw.

17. coronary*.tw.

18. angina*.tw.

19. myocard*.tw.

20. isch? em*.tw.

21. arrhythmi*.tw.

22. tachycardi*.tw.

23. fibrillat*.tw.

24. or/13-23

25. 12 and 24

26. random \$.tw.

27. factorial\$.tw.

28. crossover\$.tw.

29. cross-over\$.tw.

30. placebo $\$$.tw.

31. (doubl\$ adj blind\$).tw.

32. (singl\$ adj blind $\$$ ).tw.

33. assign $\$$.tw.

34. allocat\$.tw.

35. volunteer\$.tw.

36. control*.tw.

37. "2000“.md.

38. or/26-37

39. 25 and 38

\section{CPCI-S}

\# 11 \#10 AND \#9

\# 10 TS=(random* or blind* or allocat* or assign* or trial* or placebo* or crossover* or cross-over*)

\# 9 \#8 AND \#7

\# 8 TS $=\left(\right.$ cardio* $^{*}$ or cardia* or heart* or coronary* or angina* or myocard* or ischem* or ischaem* or arrhythmi* or tachycardi* or fibrillat*)

\# 7 \#6 OR \#5 OR \#4 OR \#3 OR \#2 OR \#1

\# 6 TS $=\left(\left(\right.\right.$ sex* $^{*}$ or intima* $)$ near/4 (relation* or spouse* or partner $\left.\left.^{*}\right)\right)$

\# 5 TS $=\left(\left(\operatorname{sex}^{*}\right.\right.$ or erect* or libido) near/2 (function* or dysfunction* ${ }^{*}$ or disorder*) $)$

\# 4 TS $=\left(\right.$ sex $^{*}$ near/4 (interest* or wish* $^{*}$ or activ* or behav* or libido))

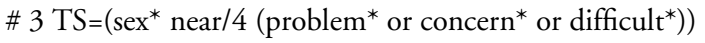

\# 2 TS=(sex* near/4 (help* or advi* or inform* or guid* or skill*))

Sexual counselling for sexual problems in patients with cardiovascular disease (Review)

Copyright @ 2016 The Cochrane Collaboration. Published by John Wiley \& Sons, Ltd. 
\# $1 \mathrm{TS}=\left(\right.$ sex $^{*}$ near $/ 4$ (counsel* ${ }^{*}$ or psycholog* ${ }^{*}$ therap*)

\section{CONTRIBUTIONSOFAUTHORS}

MB had the original idea for this Cochrane review and was the lead author of the Cochrane protocol, Byrne 2014, and review. MB is the primary contact author and guarantor for this Cochrane review.

$\mathrm{MB}$ and SD screened the literature search results and extracted data from the included trials.

DD contributed review methodology expertise to the design, conduct, and reporting of the protocol and review. DD acted as a third review author where needed.

All review authors contributed important intellectual content throughout the review process, and contributed to writing and editing the final protocol and review.

\section{DECLARATIONSOF INTEREST}

$\mathrm{MB}, \mathrm{SD}, \mathrm{BF}, \mathrm{JM}, \mathrm{TJ}$ and DD have no known conflicts of interest.

All review authors, except DD, are published researchers in the area of sexual counselling and cardiovascular disease.

One review author, ES, authored one of the included studies (Steinke 2004). We paid special attention to ensure accurate assessment of eligibility and risk of bias of this study and DD, who was not involved in the study, reviewed the process. ES has no other known conflicts of interest.

\section{SOURCES OF SUPPORT}

\section{Internal sources}

- No sources of support supplied

\section{External sources}

- This project was supported by the National Institute for Health Research via Cochrane Infrastructure funding to the Cochrane Heart Group. The views and opinions expressed therein are those of the review authors and do not necessarily reflect those of the Systematic Reviews Programme, NIHR, the NHS, or the Department of Health, UK.

- The Cochrane Heart Group US Satellite is supported by intramural support from the Northwestern University Feinberg School of Medicine and the Northwestern University Clinical and Translational Science (NUCATS) Institute (UL1TR000150), USA.

\section{DIFFERENCES BETWEEN PROTOCOLANDREVIEW}

In the Cochrane protocol, under the heading of 'Searching other resources', we proposed that we would "search reference lists of eligible papers and reviews" and "contact the principal investigators of identified studies to ascertain if they are aware of any other relevant published or unpublished studies in the area" (Byrne 2014). We did not conduct these additional searches of other resources as part of the review process. We, as review authors who are internationally experienced in this field, considered it highly likely that we had captured all ongoing intervention research activity via the core search strategies.

We reported one outcome (knowledge about sex after a MI) in the review that we had not included in the protocol. In addition, we expanded the secondary outcome stated in the Cochrane protocol as 'Quality of life or psychological well-being' within the review to include two separate outcomes: 'quality of life' and 'psychological well-being, including depression and anxiety'. Also we added an additional example of a psychological well-being tool to the outcomes list, namely Speilberger's State-Trait Anxiety Inventory (Speilberger 1983). Anxiety is a core component of psychological well-being and we should have specified it in the Cochrane protocol.

Sexual counselling for sexual problems in patients with cardiovascular disease (Review) 
We included a 'Summary of findings' table and performed GRADE assessments in this Cochrane review, which we had not stated in the protocol.

\section{NDEX TERMS}

\section{Medical Subject Headings (MeSH)}

*Sex Counseling; Cardiovascular Diseases [*complications; rehabilitation]; Coronary Artery Bypass; Myocardial Infarction [complications; rehabilitation]; Patient Satisfaction; Randomized Controlled Trials as Topic; Sexual Dysfunction, Physiological [psychology; *rehabilitation]; Sexual Partners

\section{MeSH check words}

Female; Humans; Male 